\title{
Enhancing Spinal Plasticity Amplifies the Benefits of Rehabilitative Training and Improves Recovery from Stroke
}

\author{
Anna M. Wiersma, ${ }^{1}$ Karim Fouad, ${ }^{1,2}$ and ${ }^{-I a n}$ R. Winship ${ }^{1,3}$ \\ ${ }^{1}$ Neuroscience and Mental Health Institute, ${ }^{2}$ Faculty of Rehabilitation Medicine, and ${ }^{3}$ Neurochemical Research Unit, Department of Psychiatry, Faculty of \\ Medicine and Dentistry, University of Alberta, Edmonton, Alberta T6G 2G3, Canada
}

The limited recovery that occurs following stroke happens almost entirely in the first weeks postinjury. Moreover, the efficacy of rehabilitative training is limited beyond this narrow time frame. Sprouting of spared corticospinal tract axons in the contralesional spinal cord makes a significant contribution to sensorimotor recovery, but this structural plasticity is also limited to the first few weeks after stroke. Here, we tested the hypothesis that inducing plasticity in the spinal cord during chronic stroke could improve recovery from persistent sensorimotor impairment. We potentiated spinal plasticity during chronic stroke, weeks after the initial ischemic injury, in male Sprague-Dawley rats via intraspinal injections of chondroitinase ABC. Our data show that chondroitinase injections into the contralesional gray matter of the cervical spinal cord administered $28 \mathrm{~d}$ after stroke induced significant sprouting of corticospinal axons originating in the peri-infarct cortex. Chondroitinase $\mathrm{ABC}$ injection during chronic stroke without additional training resulted in moderate improvements of sensorimotor deficits. Importantly, this therapy dramatically potentiated the efficacy of rehabilitative training delivered during chronic stroke in a skilled forelimb reaching task. These novel data suggest that spinal therapy during chronic stroke can amplify the benefits of delayed rehabilitative training with the potential to reduce permanent disability in stroke survivors.

Key words: chondroitin sulfate proteoglycans; chondroitinase $\mathrm{ABC}$; corticospinal tract; rehabilitation; sensorimotor cortex; skilled reaching

\section{Significance Statement}

The brain and spinal cord undergo adaptive rewiring ("plasticity") following stroke. This plasticity allows for partial functional recovery from stroke induced sensorimotor impairments. However, the plasticity that underlies recovery occurs predominantly in the first weeks following stroke, and most stroke survivors are left with permanent disability even after rehabilitation. Using animal models, our data show that removal of plasticity-inhibiting signals in the spinal cord (via intraspinal injections of the enzyme chondroitinase $\mathrm{ABC}$ ) augments rewiring of circuits connecting the brain to the spinal cord, even weeks after stroke. Moreover, this plasticity can be harnessed by rehabilitative training to significantly promote sensorimotor recovery. Thus, intraspinal therapy may augment rehabilitative training and improve recovery even in individuals living with chronic disability due to stroke.

\section{Introduction}

Although the CNS possesses endogenous mechanisms for plasticity within spared circuits that enables partial recovery of function lost to brain damage (Murphy and Corbett, 2009; Winship

\footnotetext{
Received March 21, 2017; revised Sept. 15, 2017; accepted 0ct. 1, 2017.

Author contributions: A.M.W., K.F., and I.R.W. designed research;A.M.W. performed research; A.M.W. and I.R.W. analyzed data; A.M.W., K.F., and I.R.W. wrote the paper.

This work was supported by Alberta Innovates Health Solutions, Heart and Stroke Foundation of Canada, Canadian Institutes of Health Research and the Natural Sciences, and Engineering Research Council of Canada (I.R.W.), and grants from the Canada Foundation for Innovation and Province of Alberta Small Equipment Grants (I.R.W.) were used to acquire confocal microscopy equipment.

The authors declare no competing financial interests.

Correspondence should be addressed to Dr. lan R. Winship, University of Alberta, Department of Psychiatry, 12-127 Clinical Sciences Building, Edmonton, AB T6G 2G3, Canada. E-mail: iwinship@ualberta.ca.

DOI:10.1523/JNEUROSCI.0770-17.2017

Copyright $\odot 2017$ the authors $\quad 0270-6474 / 17 / 3710983-15 \$ 15.00 / 0$
}

and Murphy, 2009; Overman and Carmichael, 2014), recovery after stroke is limited and most stroke survivors are left with persistent sensorimotor disability (Dobkin, 2008; Kessner et al., 2016). This incomplete recovery occurs almost exclusively in the first few weeks following the stroke: in rodent models, recovery plateaus after $\sim 4$ weeks (Sist et al., 2014; Wahl and Schwab, 2014; Wahl et al., 2014; Fig. 1A), whereas human stroke survivors complete most of their recovery within the first 3 months after stroke (Steinberg and Augustine, 1997; Green, 2003; Kitago and Krakauer, 2013). It has been suggested that the type and timing of rehabilitative training is a principal determinant of the degree of recovery (Krakauer et al., 2012; Wahl and Schwab, 2014). However, the optimal period for restoration of function appears to be finite. After this window, recovery stagnates and further rehabilitation has limited efficacy (Dimyan and Cohen, 2011). Moreover, nu- 
A
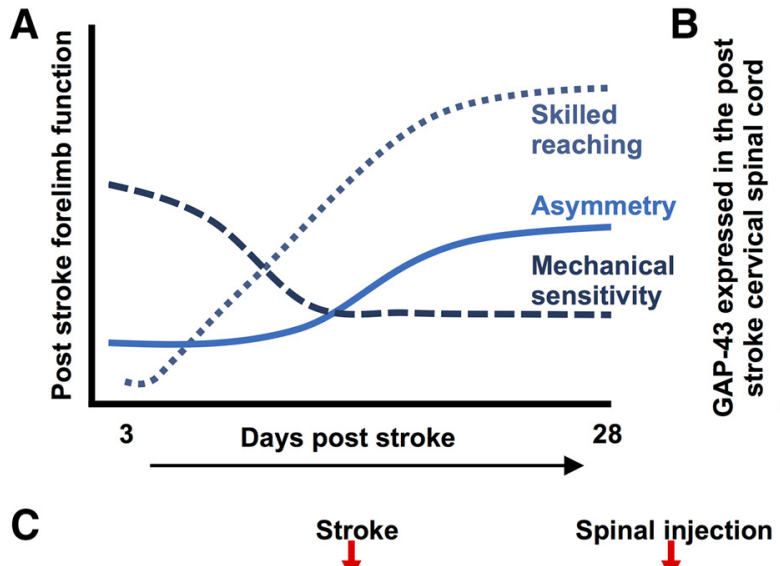

C Stroke Spinal injection

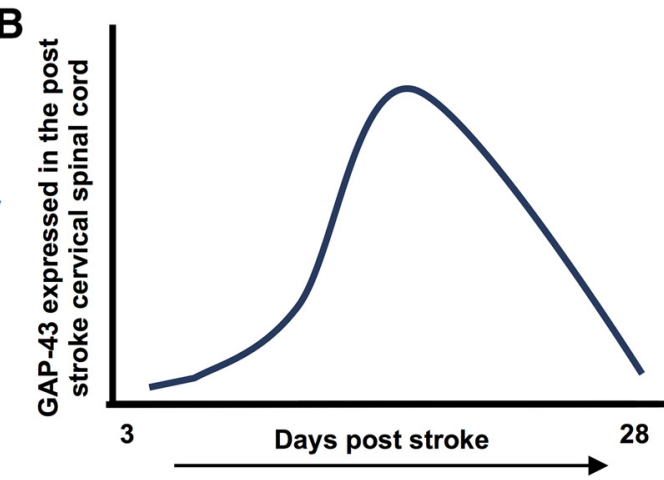

Cortical tracer

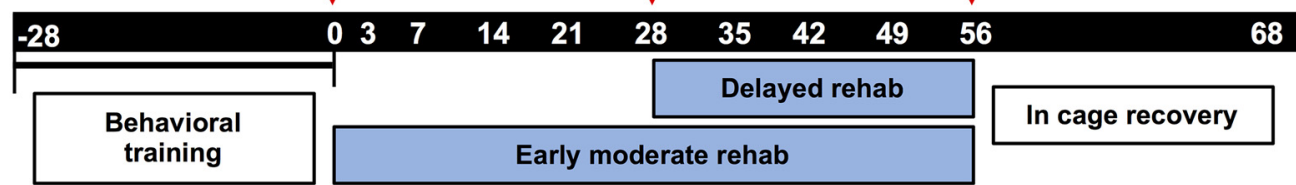
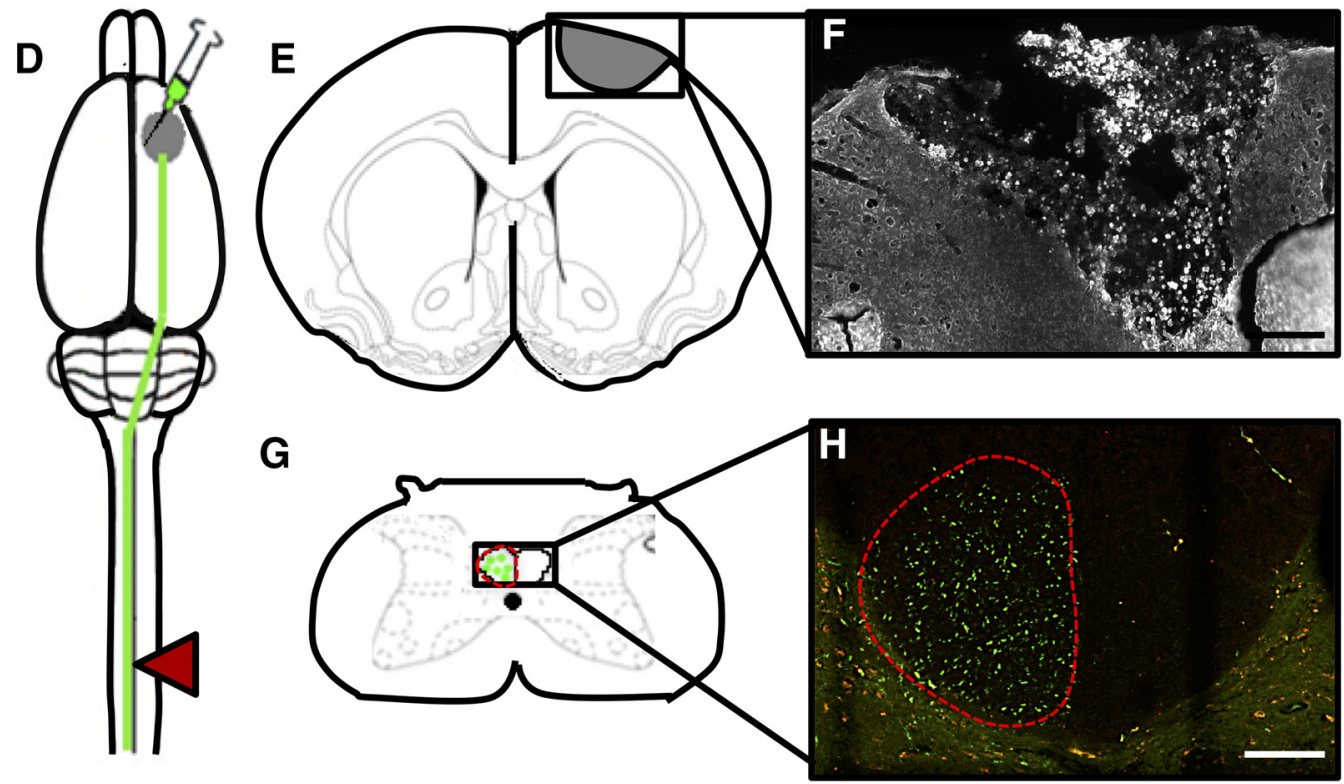

Figure 1. Experimental design. $\boldsymbol{A}$, Stroke induces sensorimotor impairment in rats that recover over the weeks following injury. Skilled reaching recovers over the first 4 weeks, but plateaus after this point. Spontaneous forelimb use preference shows a similar time course, with no further recovery after 4 weeks. If cortical lesions include the somatosensory cortex, mechanical sensitivity is impaired and shows partial recovery by 4 weeks. $\boldsymbol{B}$, The time course of post-stroke recovery corresponds with relative changes in cervical spinal cord protein expression associated with plasticity and nerve regrowth promotion. Periods of heightened sensorimotor recovery coincide with periods of peak expression of trophic factor GAP-43 in the spinal cord. Plateaued recovery coincides with a return to baseline levels of this molecule (Sist et al., 2014). Our experiments were designed to test the hypothesis that spinal microinjection of ChABC can augment plasticity of the corticospinal tract and improve recovery even during chronic stroke, after this period of heightened plasticity has ended. $C$, The experimental timeline of task-specific training and testing and the surgical procedures used to test this hypothesis. D, Schematic illustrating targeted ischemic stroke over the forelimb sensorimotor cortex (gray circle), the location of anterograde tracer injection medial to the ischemic infarct to label axons descending in the corticospinal tract (green line), and the location of spinal injection of ChABC or Pen (control enzyme) at $28 \mathrm{~d}$ post-stroke (red triangle). $\boldsymbol{E}$, Illustration of coronal section ( +1.00 anterior of bregma) demonstrating average size and stereotactic location of induced ischemic lesion. $\boldsymbol{F}$, Representative confocal differential interference contrast image of infarct $68 \mathrm{~d}$ postinjury $(10 \times)$. Scale bar, $1000 \mu \mathrm{m}$. G, Illustration of a transverse section at cervical vertebrae 5 , with tracer labeled axons in contralesional CST. $\boldsymbol{H}$, Representative image of rat corticospinal tract axons labeled with anterograde AlexaFluor 488-tagged dextran tracers (visualized using spectral settings for the FITC emission spectrum on the confocal microscope) shown in green. Images were also collected using a Rhodamine filter set to confirm that fluorescence from axon fragments were specific to the tracer as shown in red ( $10 \times$ ). Scale bar, $2000 \mu \mathrm{m}$.

merous randomized clinical trials of rehabilitation therapies have reported neutral or negative results (AVERT Trial Collaboration Group, 2015; Winstein et al., 2016). Thus, the degree to which rehabilitative training can improve function above spontaneous biological recovery has been questioned, and improved strategies to reduce disability due to stroke are required (Lowry, 2010; Krakauer and Marshall, 2015).
Augmenting plasticity of spared neural circuits can improve recovery from CNS injury. Experimental plasticity-enhancing treatments are most effective when delivered early after CNS injury and accompanied by rehabilitative training (van den Brand et al., 2012; Hara, 2015; Jones and Adkins, 2015). Notably, plasticity of the corticospinal tract (CST) originating in the uninjured contralesional motor cortex makes a significant contribution to 
recovery from stroke-induced sensorimotor impairment (Ueno et al., 2012; Silasi and Murphy, 2014; Wahl and Schwab, 2014; Wahl et al., 2014). However, the spinal plasticity elicited by cortical stroke has a finite temporal window and sensorimotor recovery plateaus after this period (Sist et al., 2014; Kessner et al., 2016; Fig. 1A,B).

One approach to augment plasticity is to manipulate chondroitin sulfate proteoglycans (CSPGs) in the extracellular matrix. These molecules are potent inhibitors of neurite growth, inducing growth cone collapse, sterically hindering dendritic rearrangement, and physically blocking growth promoting adhesion molecules (Silver and Miller, 2004; Afshari et al., 2010; Cua et al., 2013). CSPGs also interact with tyrosine phosphate (Shen et al., 2009) and related leukocyte common antigen-related phosphatase receptors (Xu et al., 2015) to induce growth inhibitory signaling. Modulation of CSPGs and their signaling pathways can induce in increased axonal growth after injury (Monnier et al., 2003; Fisher et al., 2011; Sharma et al., 2012). Digestion of CSPGs with the bacterial enzyme chondroitinase ABC (ChABC) augments structural plasticity (Moon et al., 2001; Houle et al., 2006; Maier and Schwab, 2006; Burnside and Bradbury, 2014). ChABC injection into the spinal cord during the period of heightened spinal plasticity that follows stroke significantly plasticity of the uninjured CST and improves sensorimotor recovery (Soleman et al., 2012). However, it is not known whether such spinal therapies can be used during chronic stroke to reduce disability. Here, we assessed whether spinal delivery of ChABC during chronic stroke enhance plasticity of the CST originating in peri-infarct cortex and potentiate task-specific rehabilitative training (Fig. 1C). Although previous studies suggest that intraspinal ChABC delivered during the subacute period after stroke can induce plasticity of the uninjured CST (Soleman et al., 2012; Wahl et al., 2014), these studies did not evaluate the potentially important contribution of CST projections from the spared cortex adjacent the infarct and did not evaluate the interaction between rehabilitative training and plasticity-enhancing therapy. Our findings demonstrate that removing inhibition of axonal growth by CSPGs in the spinal cord induces structural plasticity of the spared CST and facilitates rehabilitative training even during chronic stroke.

\section{Materials and Methods}

\section{Animals}

Male Sprague-Dawley rats (400-550 g; Charles River Laboratories) were housed in pairs (2 per cage) under standard laboratory conditions $(12 \mathrm{~h}$ light/dark cycles). Water and standard laboratory rat chow were available ad libitum, except during the training period when feeding was reduced to $50 \mathrm{~g}$ daily to maintain $85-90 \%$ ad libitum feeding body weight. All animal protocols were approved by the University of Alberta Animal Care and Use Committee in accordance with Canadian Council on Animal Care guidelines.

\section{Experimental design}

The general experimental design is shown in Figure 1. The first cohort of rats $(n=40)$ was divided into four experimental groups: Animals received photothrombotic stroke with a delayed spinal cord injection of ChABC enzyme $(n=11)$ or the control enzyme penicillinase (Pen; $n=$ $11)$ or sham-stroke with delayed injections of ChABC $(n=9)$ or Pen $(n=9)$. A second cohort of rats $(n=24)$ received photothrombotic strokes and injection of ChABC $(n=12)$ or Pen $(n=12)$ on post-stroke day 28 followed by either delayed high-intensity rehabilitative training in a forelimb reaching task ( $n=6$ per group) or delayed moderate intensity training ( $n=6$ per group). Finally, a third cohort of rats received photothrombotic stroke $(n=16)$ and moderate reaching training that was initiated $3 \mathrm{~d}$ after stroke and continued until spinal injections of ChABC $(n=8)$ or Pen $(n=8)$ on day 28. Following injection, training recom- menced for an additional $28 \mathrm{~d}$. Animals were randomly assigned to surgical groups. Following surgery, animals were randomly assigned a number code to ensure all experimenters were blind to treatment groups throughout behavioral assessment. At the conclusion of the experiment, extracted brains were assigned a letter code to ensure that the experimenter was blind to surgical and rehabilitative training groups. The animal number and letter codes were not decoded until all testing and analyses were completed.

\section{Surgical procedures}

Surgical procedures were performed in animals deeply anesthetized with $2-2.5 \%$ isoflurane (in 70\% nitrous oxide and 30\% oxygen at a flow rate of $1 \mathrm{~L} / \mathrm{min}$ ). Body temperature was maintained at $37^{\circ} \mathrm{C}$ with a rectal temperature probe and heating pad.

\section{Photothrombotic stroke}

The forelimb sensorimotor cortex corresponding to the preferred limb in skilled reaching tasks was located using stereotaxic coordinates (1-4 mm lateral; -1 to $+3 \mathrm{~mm}$ anterior of bregma; Watson and Paxinos, 2006). Skull over this cortex was thinned followed by a tail vein injection of Rose Bengal (30 mg/kg in $0.01 \mathrm{~m}$ sterile PBS; Sigma-Aldrich). The cortex was illuminated using a collimated beam of green laser light $(532 \mathrm{~nm}, 17 \mathrm{~mW}$; $\sim 4.0 \mathrm{~mm}$ in diameter) for $10 \mathrm{~min}$ to induce photothrombosis. Sham-stroke controls underwent identical procedures without skull illumination.

\section{Spinal administration of ChABC}

$\mathrm{ChABC}$ was delivered unilaterally to the cervical spinal cord contralateral to the stroke. Briefly, a partial laminectomy of C4 and C5 was performed to expose the contralesional spinal cord. Using a $10 \mu$ l Hamilton syringe mounted on a stereotaxic arm and fitted with a pulled glass tip, a $1.0 \mu \mathrm{l}$ injection of either chondroitinase ABC ( $10 \mathrm{U} / \mathrm{ml}$; Sigma-Aldrich) or Pen [same weight $(\mu \mathrm{g})$ per volume $(\mathrm{ml})$ as ChABC; Sigma-Aldrich] was injected $(0.2 \mu \mathrm{l} / \mathrm{min})$ at both $\mathrm{C} 4$ and $\mathrm{C} 5$. Injections were positioned to $1 \mathrm{~mm}$ lateral of the midline at a depth of $1-1.5 \mathrm{~mm}$. The pipette was left in place for $1 \mathrm{~min}$ after injection to reduce backflow.

\section{Anterograde anatomical tracing of the corticospinal tract}

AlexaFluor 488-tagged dextran tracers were injected into peri-infarct motor cortex areas to label CST axons projecting to the cervical spinal cord. Two burr holes were made $1 \mathrm{~mm}$ medial to the ischemic lesion using stereotactic coordinates at $1 \mathrm{~mm}$ lateral of the midline and $1.5 \mathrm{~mm}$ anterior of bregma and $1 \mathrm{~mm}$ lateral of the midline and $-0.5 \mathrm{~mm}$ anterior of bregma (Fig. $2 D-F$ ). Using a Hamilton syringe fitted with a pulled glass microtubule tip, $1 \mu \mathrm{l}(0.2 \mu \mathrm{l} / \mathrm{min})$ of Dextran, AlexaFluor 488 ; 10,000 MW ( $1 \mathrm{mg} / \mu$ l; Life Technologies, D-22910) was injected $1.5 \mathrm{~mm}$ below the cortical surface at each location.

\section{Transcardial perfusion and tissue preparation}

At $68 \mathrm{~d}$ post-stroke rats were perfused with $250 \mathrm{ml}$ of saline heparin $(0.02 \%)$ solution $\left(37^{\circ} \mathrm{C}\right)$ followed by $250 \mathrm{ml}$ of $4 \%$ formalin solution $\left(4^{\circ} \mathrm{C}\right)$ at a flow rate of $25 \mathrm{ml} / \mathrm{min}$. Brains and spinal cords were removed and submerged in $4 \%$ liquid formalin overnight at $\left(4^{\circ} \mathrm{C}\right)$, and then transferred to $30 \%$ sucrose solution $\left(4^{\circ} \mathrm{C}\right)$ until they lost buoyance.

\section{Stroke volume and tracer injection analysis}

Brains were flash frozen and cryosectioned in $20 \mu \mathrm{m}$ coronal slices. Infarct areas were determined in ImageJ for every slice spanning the lesion and integrated to determine the total stroke volume for each animal. Animals with stroke volume $<2 \mathrm{~mm}^{3}$ were not included in the study $(n=1)$. The location and depth corresponding to the center of the tracer injections were determined by examining each coronal section under fluorescent microscopy with a Leica DMI6000B inverted microscope. Cortical maps showing the topographical location of induced stroke and tracer injections were created for each animal by recording the stereotaxic coordinate of stroke boundaries on each coronal slice of tissue spanning the lesion (Fig. 2D-F). The average location of tracer injections was also obtained by averaging the stereotaxic coordinates of all injection sites (Fig. 2G). Coronal sections for each animal at $-1.0,0.0,1.0$, and 2.0 from bregma were analyzed for depth of stroke and tracer injection (Fig. $2 \mathrm{H}$ ). The average stroke and tracer injection depth were obtained by averaging the depths from each animal. 

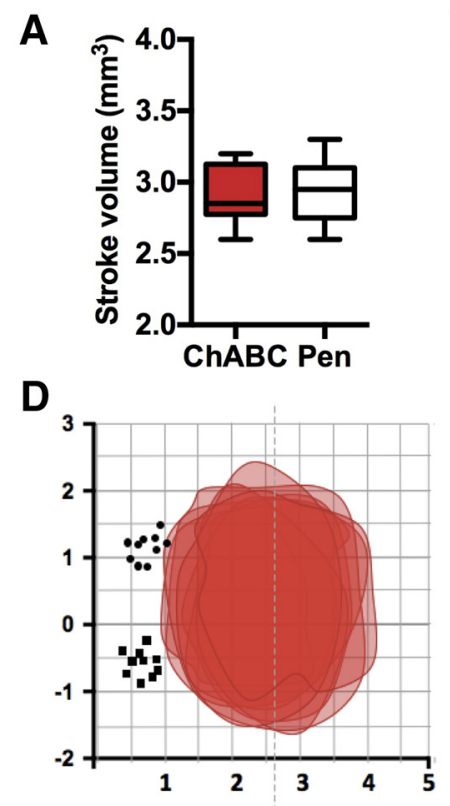

E

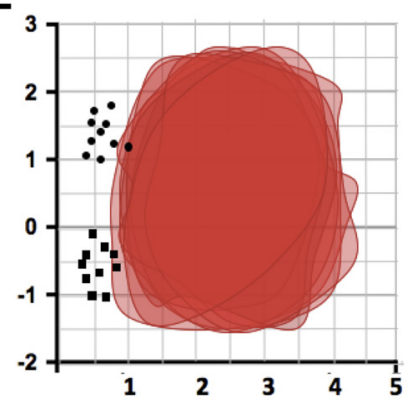

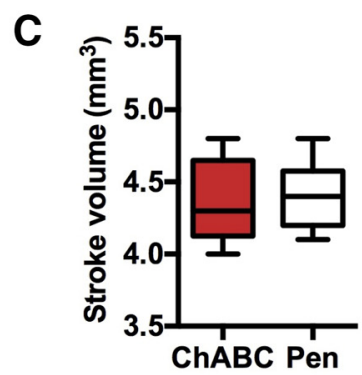

$\mathbf{F}$

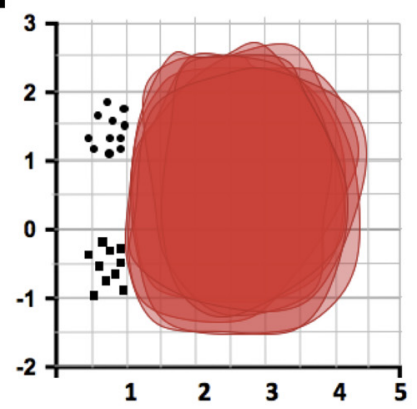

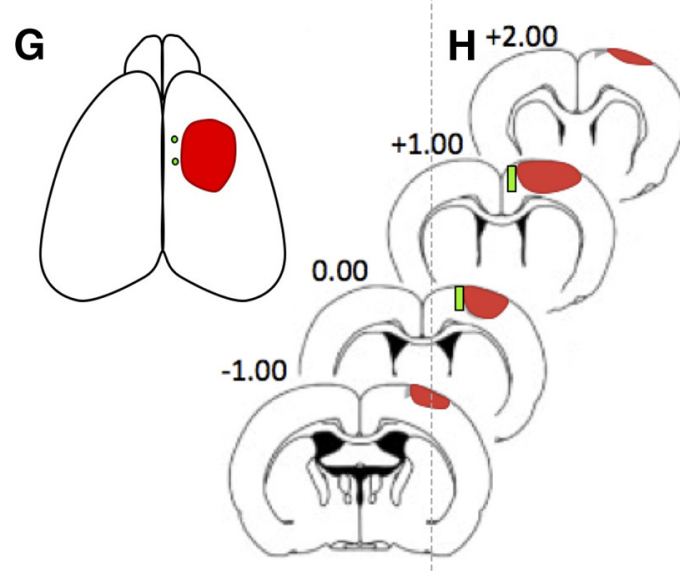
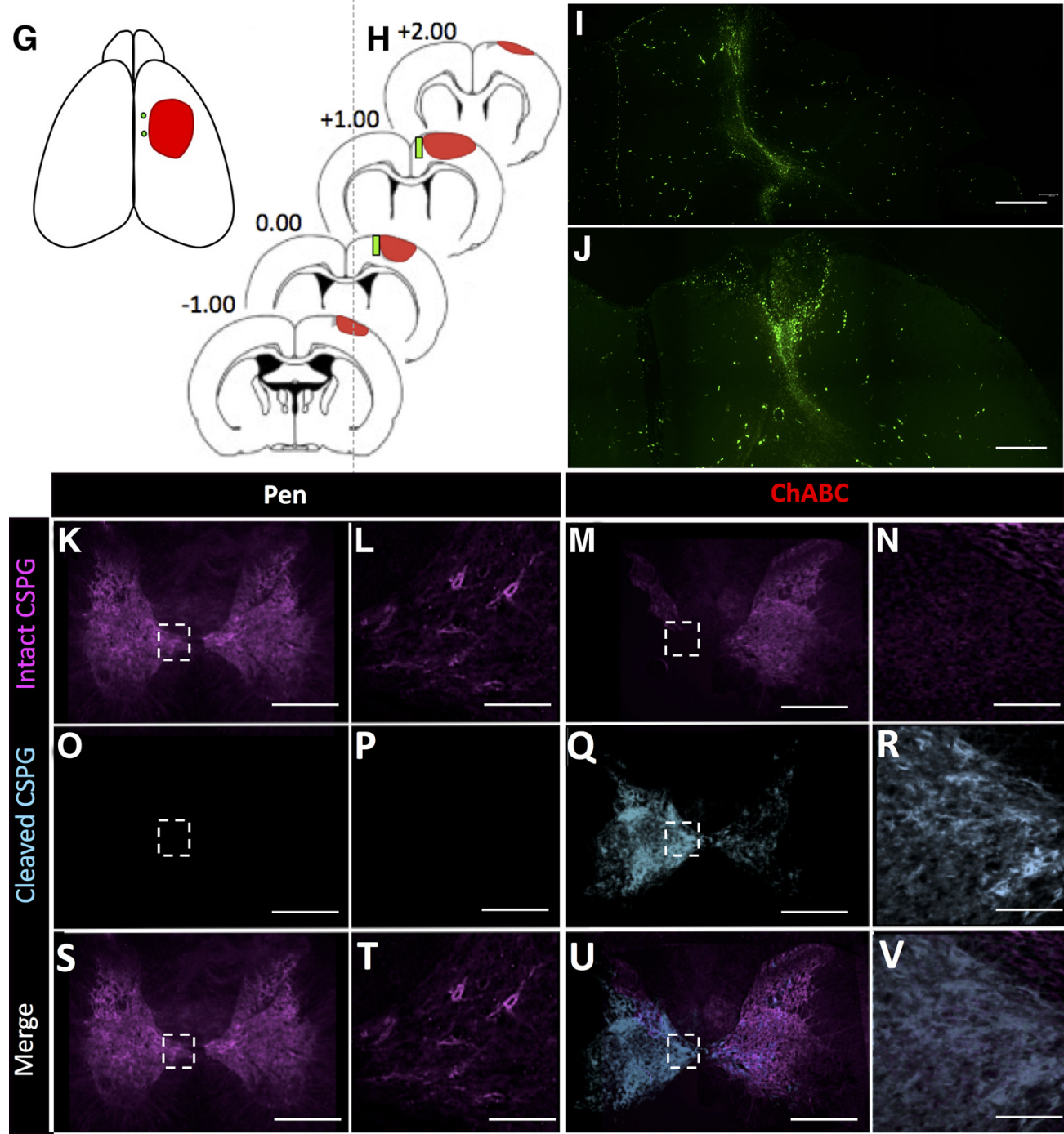

Figure 2. Stroke volumes and location of stroke and anterograde tracer injections. Average stroke size of animals treated with ChABC or Pen receiving $(\boldsymbol{A})$ no rehab, $(\boldsymbol{B})$ delayed rehab, or $(\boldsymbol{C})$ early rehab. $A$, There were no significant difference in the stroke volumes between ChABC-treated or Pen control groups when receiving no rehabilitative training (two-tailed $t$ test; $p=0.92, t=0.10$, $\mathrm{df}=18),(\boldsymbol{B})$ delayed rehabilitative training $(p=0.78, t=0.28, \mathrm{df}=14),(\boldsymbol{C})$ or early rehabilitative training $(p=0.67, t=0.44, \mathrm{df}=14) . \boldsymbol{D}-\boldsymbol{F}$, Cortical map showing the topographical location of induced stroke and tracer injections of 10 animals from each of the $(\boldsymbol{D})$ no rehab, $(\boldsymbol{E})$ delayed rehab, and $(\boldsymbol{F})$ early rehab groups. $\boldsymbol{G}$, Topographical cortical map (Figure legend continues.) 


\section{Spinal CSPG digestion}

To confirm the digestion of CSPGs in the cervical spinal cord following injection of ChABC, standard protocols were used to fluorescently label both intact CSPGs and stubs left by their enzymatic digestion. Full-length undigested CSPGs were labeled with a Biotinylated Wisteria floribunda lectin (WFA) antibody (Soleman et al., 2012; Vector Laboratories, cata$\log$ \#B-1355;1:1000) and streptavidin AlexaFlour 647 secondary antibody (Life Technologies, Catalog \#S32357: 1:400). Digested CSPG cleavage stubs were labeled with mouse anti-chondroitin-4-sulfate antibody (MP Biomedical, catalog \#0863651; 1: 1000) and donkey antimouse AlexaFlour 488 secondary antibody (Life Technologies, catalog \#S32357; 1:200). Slides were mounted with Vectashield fluorescent mounting medium containing DAPI (Vector Laboratories, catalog \# H-1500).

\section{Neuroanatomical tracer quantification}

Given the degradation of CSPGs in the cervical spinal gray matter by ChABC, we hypothesized that local increases in axonal sprouting would be reflected by increased number and length of axon fragments in the gray matter in treated animals relative to controls. To quantify this, spinal cord tissue from C3-C7 was cryosectioned in $30 \mu \mathrm{m}$ transverse slices and mounted on Superfrost Plus Gold slides (Fisher Scientific) with Fluoromount-G (Southern Biotech) mounting medium. Images were collected on a Leica SP5 Confocal microscope using a Leica HCX PL APO L $20 \times 1.0$ NA water emersion objective lens and Leica LAS AF Software. Thirty micrometer $z$-stacks ( 100 steps at $0.3 \mu \mathrm{m}, 736 \times 736 \mu \mathrm{m}$ image plane) were collected and maximum projections of all the optical planes were created (i.e., each maximum projection collapses the fibers imaged over the $30 \mu \mathrm{m}$ section into a single image). Tile scans were used to image the gray matter across both the ventral and dorsal horns and the CST. Images were coded and presented to the experimenter in random order to ensure the experimenter was blinded to experimental group. The number of labeled fibers in the CST of each animal in the most rostral section of $\mathrm{C} 4$ was counted by the experimenter using WCIF-ImageJ. Next, the experimenter counted the number of projections into the gray matter on both the contralesional and ipsilesional side of the spinal cord; these counts were repeated on 20 spinal cord sections of $30 \mu \mathrm{m}$ thickness, spaced $210 \mu \mathrm{m}$ apart (every 8 slices), spanning $5000 \mu \mathrm{m}$ between $\mathrm{C} 4$ and C5, and the average number of axon segments on each side of the gray matter was determined. The experimenter also measured the length of each axon segment $>1 \mu \mathrm{m}$ using ImageJ. The total length of axon segments in the gray matter was summed and divided by the number of traced axons counted in the CST at C4 to reduce variability due to inter-animal differences in tracer efficacy or uptake. This axon length analysis was repeated on 20 sections and an average normalized length was determined for each animal. Stereological quantification of fiber density was per-

\section{$\leftarrow$}

(Figure legend continued.) showing the average location and area of stroke across all experimental groups and the average location of the two tracer injections. $\boldsymbol{H}$, Stack of coronal brain sections showing the average location and depth of stroke and injected cortical tracers. $I, J$, Representative images of the anterograde Alexafluor 488-tagged tracer cortical injection sites medial to stroke lesion, tracer in green (visualized using spectral settings for the FITC emission spectrum on the confocal microscope; $10 \times$ ). Scale bars, $2000 \mu \mathrm{m}$. $\boldsymbol{K}-\boldsymbol{N}$, Spinal cord cross sections showing full-length or undigested CSPGs labeled with WFA and AlexaFluor 647 (visualized using spectral settings for the $\mathrm{CY} 5$ emission spectrum on the confocal microscope) in purple (K) Pen-treated and (M) ChABC-treated animals $56 \mathrm{~d}$ after injection $(5 \times)$. Scale bar, $4000 \mu \mathrm{m}$. L, N, High-magnification images (20X; scale bar. $200 \mu \mathrm{m}$ ) of WFA with AlexaFluor 647 in purple from area delineated with white boxes in $\boldsymbol{K}$ and $\boldsymbol{M}$. $\mathbf{0}-\boldsymbol{R}$, Spinal cord cross sections showing digested CSPG stubs labeled with anti-chondroitin-4-sulfate and AlexaFluor 488 (visualized using spectral settings for the FITC emission spectrum on the confocal microscope) in blue of ( $\mathbf{O})$ Pen-treated and ( $(\mathbf{Q})$ ChABC-treated animals 56 d after injection ( $5 \times)$ Scale bar, 4000 $\mu \mathrm{m} . \boldsymbol{P}, \boldsymbol{R}$, High-magnification images ( $20 \times$; scale bar, $200 \mu \mathrm{m}$ ) of anti-chondroitin-4-sulfate and Alexafluor 488 in blue from area delineated with white boxes in $\mathbf{O}$ and $\mathbf{Q}$. S-V, Merged images of undigested CSPG labeled with WFA with AlexaFluor 647 in purple and digested CSPG stubs labeled with anti-chondroitin-4-sulfate and AlexaFluor 688 in blue from $(\boldsymbol{K}, \boldsymbol{O})$ Pen and $(\boldsymbol{M}, \mathbf{Q}) \mathrm{ChABC}$-treated animals at $5 \times$ and $20 \times$ magnification. Scale bars: $\boldsymbol{S}, \boldsymbol{U}, 4000 \mu \mathrm{m}$; $T, V, 200 \mu \mathrm{m}$. formed using a $50 \times 50$ grid fitted over the gray matter. Dorsal to ventral lines were positioned from the tip of lamina 1 to the border of ventral horn gray mater, and medial to lateral lines where positioned from the midline the lateral gray matter border. This grid was custom fit over the gray matter to ensure that variation in tissue shrinkage, orientation and deformation would not affect the total fiber counts. The number of fibers $>5 \mu \mathrm{m}$ in each grid section was counted to determine the density and distribution of CST axons in the gray matter.

\section{Serotonin immunohistochemistry and densitometric quantification}

Standard immunofluorescence protocols were used to label serotoninergic fibers in the cervical spinal cord using a primary rabbit anti-serotonin antibody in (Sigma-Aldrich, catalog \#5545; 1:500) and goat anti-rabbit Texas Red secondary antibody (Vector Laboratories, catalog \#TT-1000; 1: 500). Slides were mounted with Vectashield fluorescent mounting medium containing DAPI (Vector Laboratories, catalog \#H-1500). Densitometric quantification of serotonergic axon density was performed on regions-of-interest in the ventral horn on both the contralesional and ipsilesional side (in 5 tissue slices per animal; see Fig. $8 B$ ) using a Leica DMI6000B inverted microscope (at $10 \times$ magnification). ImageJ software was used to quantify optical density.

\section{Skilled reaching tasks}

On skilled reaching tasks, rats were trained twice per day (with 4 h between sessions) on 28 consecutive days and baseline values of performance were determined from performance over $3 \mathrm{~d}$ before stroke. Testing consisted of two test sessions, $4 \mathrm{~h}$ apart, with the average scores for the two tests reported. All figures report the reaching success rate normalized to baseline before stroke.

Montoya staircase task. Rats were placed in a clear Plexiglas box with a central platform and a thin staircase with indents on each of the seven stairs ( 21 pellets per side of the animal). Training consisted of $15 \mathrm{~min}$ uninterrupted in the Montoya staircase Plexiglas box and the number of pellets (45 mg rodent pellets in banana flavor, TestDiet), which rats successfully reached and consumed was recorded. Both the left and right staircases were filled with pellets during the first 14 training sessions. Once a dominant forepaw was established, the staircase on only the dominant forelimb side of the animal was loaded with pellets. Those animals which did not meet the minimum criteria of successfully retrieving 9 of 21 pellets with either forelimb were eliminated from the study $(n=4)$.

The single pellet-reaching task. Animals were placed in a clear Plexiglas box $(39.5 \times 12.5 \times 47.5 \mathrm{~cm})$ with a $1-\mathrm{cm}$-wide slit in the front wall running floor to ceiling and a shelf $(4 \times 4 \mathrm{~cm})$ outside the box mounted $6 \mathrm{~cm}$ above the mesh floor. Pellets were placed $1.5 \mathrm{~cm}$ from the interior wall on the shelf outside the slit. Rats reached for pellets through the slit and were trained to return to the back of the cage between each successful pellet retrieval. During training, animals were allowed to reach for a maximum of 100 pellets and those animals unable to successfully retrieve 10 pellets after $15 \mathrm{~d}$ of training were eliminated from the study $(n=11)$. After the first 15 sessions, a preferred limb was established; in all subsequent training sessions only the platform contralateral the preferred limb was loaded with pellets. The number of reaching attempts and the number of successful retrievals were tracked.

\section{Spontaneous forelimb use}

The cylinder test was used to assess spontaneous forelimb use of animals when exploring vertical surfaces. Rats were placed in a clear Plexiglas cylinder (44 cm height $\times 35 \mathrm{~cm}$ diameter) with an open top and video recorded in the cylinder for $10 \mathrm{~min}$. The first 20 rearing motions during exploration were analyzed and scored. We defined "left" or "right" forelimb contact as placement of a single forepaw against the wall of the cylinder with the whole palm to support the body weight of the animal. If the animals touched the vertical surface with both paws or with one paw quickly followed by the second paw, the touch was scored as "both". The number of contacts with the impaired limb and unimpaired limb is reported as a percentage of the total number of contacts.

\section{Mechanical sensitivity testing}

Mechanical sensitivity was assessed in the rat forepaw mid-plantar region using von Frey monofilaments. The procedure was modified for the 
forepaw from procedures using the staircase method with removal of monofilaments 9 (4.17) and 13 (4.93) as suggested by Bradman et al. (2015). The filament application was standardized to $2 \mathrm{~s}$ on all trial with 10 applications. Animals were either scored as (1) responding or (2) nonresponding for each filament application; when a $50 \%$ withdrawal response was achieved, the target force of the filament was recorded as the paw withdrawal mechanical threshold (PWMT). We ensured a consistent blind operator for every experiment and analysis was done using log stimulant values to avoid bias across the range.

\section{Rehabilitative training}

Rehabilitative motor training was conducted in the single pellet-reaching chamber. The moderate intensity animals reached for 15 min twice each day with a maximum of 100 pellets per session. In the high-intensity group, animals were allowed to reach for 1000 pellets or a maximum of $2 \mathrm{~h}$, twice per day. During these training sessions animals were allowed to reach in place and did not have to return to the back of the cage before receiving the next pellet.

\section{Statistical analysis}

Statistical analyses were performed using GraphPad Prism v7. Statistical analysis of behavioral tests was performed using two-way ANOVA. HolmSidak multiple comparisons were performed between groups when a significant main effect of treatment or a significant interaction of time $\times$ treatment was found. When there was a significant main effect of time but no significant main effect of treatment or interaction we performed Holm-Sidak's post hoc testing to identify within group differences at different time points. Fiber count and length statistical analysis was performed by two-way ANOVA testing followed by post hoc Holm-Sidak's testing. Statistical evaluation of serotonergic fibers density was performed with one-way ANOVA, followed by Holm-Sidak's post hoc to compare each treatment group to the uninjured sham group. Data are reported as mean $\pm \mathrm{SEM}$.

\section{Results}

Intraspinal $\mathrm{Ch} \mathrm{ABC}$ without training reduces sensorimotor deficits in an animal model of chronic stroke

The experimental timeline is illustrated in Figure $1 C$. Photothrombotic stroke was used to induce consistent infarcts lesioning the entire forelimb sensorimotor cortex (Fig. 2A-F). Spinal injections of $\mathrm{ChABC}$ or the inactive control enzyme Pen were administered $28 \mathrm{~d}$ following stroke. At this time point, endogenous promoters of plasticity that are upregulated early after stroke have returned to baseline, and spontaneous functional recovery (with or without rehabilitation) has plateaued (Sist et al., 2014; Kessner et al., 2016; Fig. 1A,B). Spinal injections were targeted to the deinnervated contralesional side of the spinal cord at cervical level 4 (C4), which represents a major termination point for CST axons originating in the forelimb motor cortex (Fig. 1G). Digestion of CSPGs (reduced WFA staining and persistent C-4-S fluorescence) was evident in the gray matter on the contralesional side of the spinal cord at $\mathrm{C} 4$ even $28 \mathrm{~d}$ after injection (Fig. $2 K-V$ ). Before photothrombotic stroke, animals were trained and tested on a battery of sensorimotor tasks to establish pre-stroke baseline (Fig. 3A). Animals were tested again 3, 7, 14, 28,42 , and $56 \mathrm{~d}$ post-stroke to examine the time course of recovery before and after ChABC (or control) treatment (Fig. $3 A$ ).

Photothrombosis induced clear lesions of the forelimb sensorimotor cortex that were still apparent in both treatment groups $68 \mathrm{~d}$ after stroke (Fig. $2 A, D$ ). Reaching performance in the Montoya Staircase task was reduced to $42.9 \pm 7.1 \%$ and $43.2 \pm 7.10 \%$ of pre-stroke levels at $7 \mathrm{~d}$ after injury in ChABC-treated and control animals, respectively. Rats spontaneously recovered to $62.7 \pm 4.9$ and $61.6 \pm 4.7 \%$ of pre-stroke levels (ChABC and Pen controls, respectively) by $28 \mathrm{~d}$ post-stroke (before intraspinal injections). Multivariate analysis did not reveal a significant effect of ChABC treatment in stroke animals (Fig. $3 B$ ) or sham-stroke controls (Fig. 3C). However, a significant main effect of time $\left(F_{(5,100)}=25.52, p<0.0001\right)$ was observed. Notably, ChABCtreated rats showed significant improvement in reaching performance between 28 (pre-ChABC) and $56 \mathrm{~d}$ post-stroke (Holm-Sidak, $p<0.05$ ), whereas reaching performance in control rats did not improve between 28 and $56 \mathrm{~d}$ post-stroke $(p>0.05)$.

Stroke induced forelimb use asymmetry in the cylinder task showed partial recovery by $28 \mathrm{~d}$ post-stroke then plateaued between 28 and $56 \mathrm{~d}$ (Fig. 3D). There was no effect of treatment on limb use preference $(p>0.05)$, however there was a significant main effect of time $\left(F_{(4,80)}=40.21, p<0.0001\right)$. Similarly, mechanical sensitivity was impaired by stroke with complete recovery to a plateau at $28 \mathrm{~d}$ in both treatment groups. There was a significant effect of time $\left(F_{(8,112)}=12.86, p<0.0001\right)$ and a significant interaction of time and treatment group $\left(F_{(8,112)}=\right.$ 2.38, $p=0.0249$ ), however, there was no significant difference between groups at any time point using Holm-Sidak's multiple comparisons (Fig. $3 F$ ). There was no effect of treatment or time in ChABC-treated or control rats that did not receive stroke $\left(F_{(8,112)}=0.9712, p=0.4623\right.$; Fig. $\left.3 C, E, G\right)$. Thus, ChABC administered in the spinal cord $28 \mathrm{~d}$ after stroke resulted in limited improvement of sensorimotor function relative to control animals.

\section{ChABC induces axonal sprouting during chronic stroke}

Anterogradely labeled axon fragments originating at injection sites medial to the infarct (Fig. 2D-J) were imaged in the cervical spinal cord (vertebrae C4 and C5) using confocal microscopy. These axon fragments represent projections from the CST into the spinal gray matter of the cervical spinal cord originating at surviving neurons located medial to the infarct. Compensatory sprouting of these projections may act to restore connectivity between the damaged motor cortex and the partially deinnervated spinal networks. To assess this, the number and length (normalized to the total number of labeled fibers in the descending CST in the most rostral section of C4; Fig. 4C) of labeled axon fragments derived from the CST found in the spinal gray matter was determined for each animal using a maximum projection of 100 optical planes acquired over a $30 \mu \mathrm{M}$ depth. Analyses were then repeated for 20 tissue sections, each separated by $210 \mu \mathrm{m}$, for each animal (for a sample spanning $5000 \mu \mathrm{m}$ ). In ChABCtreated rats, extensive innervation of the cervical spinal gray matter by fibers emanating from the CST was apparent (Fig. 4A). In contrast, control animals had very limited innervation of the cervical gray matter by CST fibers originating in peri-infarct cortex (Fig. 4B). Significant main effects of treatment and hemisphere were observed for the average number (two-way ANOVA, treatment: $F_{(1,32)}=17.42, p=0.0002$; hemisphere: $F_{(1,32)}=12.32$, $p=0.0014$; interaction: $\left.F_{(1,32)}=9.378, p=0.0044\right)$ and mean length of axon fragments in the spinal gray matter (treatment: $F_{(1,20)}=11.04, p=0.0034$; hemisphere: $F_{(1,20)}=7.747, p=$ 0.0115 interaction: $\left.F_{(1,20)}=5.163 p=0.0343\right)$. Post hoc comparisons revealed significantly more axon fragments (mean difference of $58.6 \pm 11.5$ fibers between groups; Holm-Sidak, $p<$ 0.0001 ) and longer axon fragments (mean difference of $29.1 \pm$ $7.4 \mu \mathrm{m}$ axon length between groups; $p=0.0038$ ) in the contralesional gray matter (Fig. 4D-G). Moreover, CST fibers crossing over from the contralesional to the ipsilesional gray matter were observed in ChABC-treated animals but not controls (Fig. 4D,E). Analysis of contralesional spinal cord showed a greater dorsal to ventral (Fig. 4F) and medial to lateral (Fig. 4G) distribution of fibers in the gray matter of ChABC-treated animals compared 
A

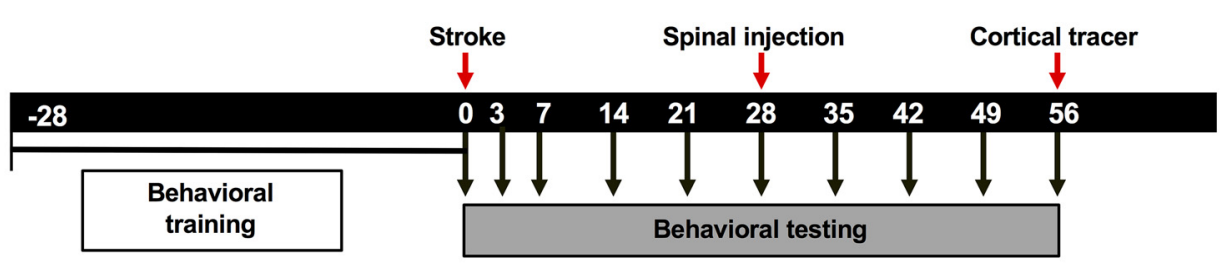

B

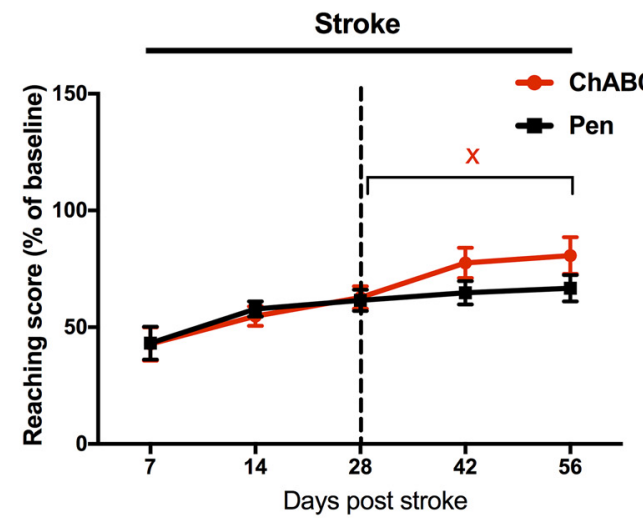

D

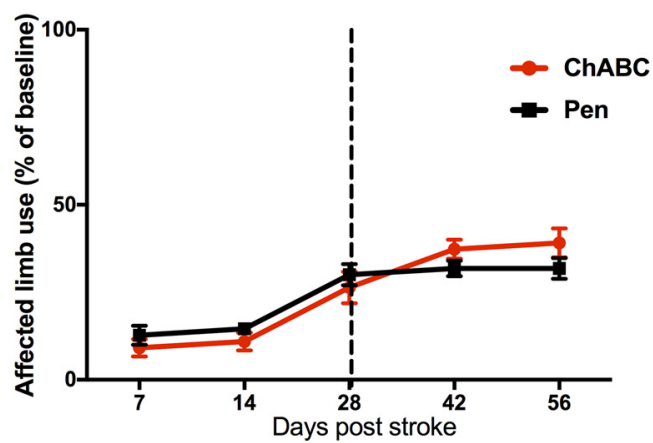

F

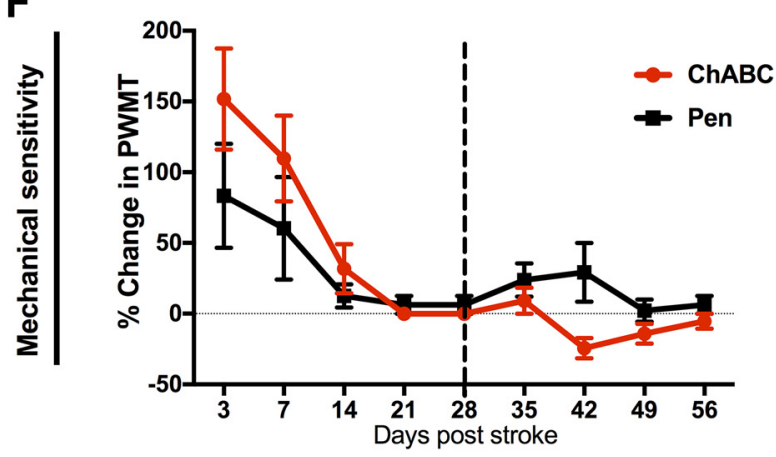

C

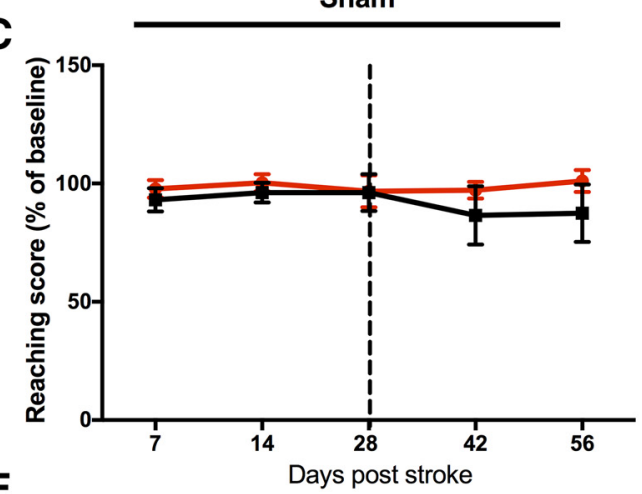

E

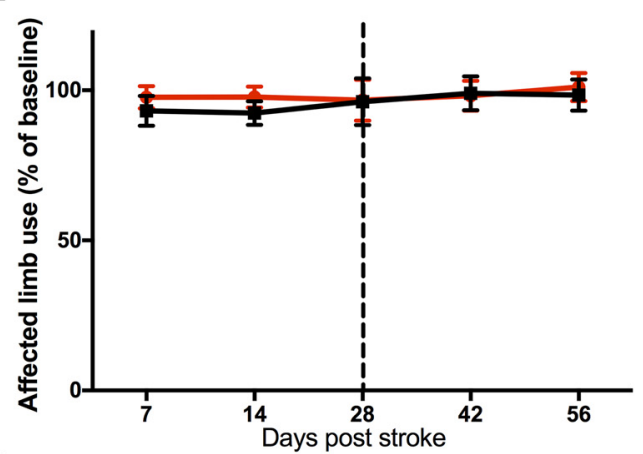

G

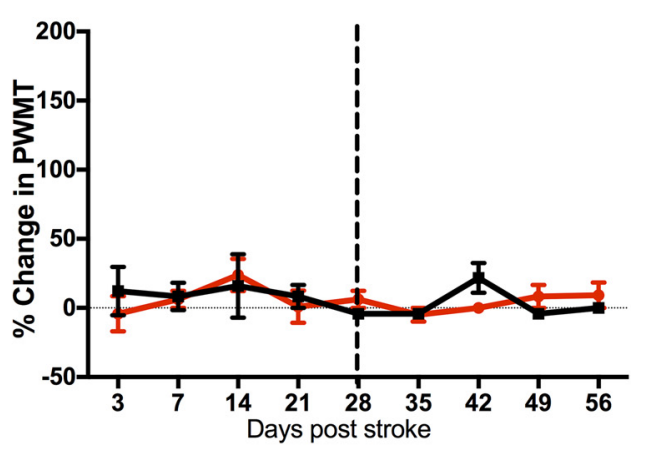

Figure 3. ChABC administered during chronic stroke without task-specific training moderately improves recovery. $\boldsymbol{A}$, Timeline showing behavioral testing and surgical procedures. $\boldsymbol{B}$, Skilled reaching in the Montoya Staircase task. There was no significant main effect of $\mathrm{ChABC}$ (relative to Pen controls) or time $\times$ treatment interaction $(p>0.05)$; however, a significant effect of time was detected $(p<0.0001)$. Holm-Sidak post hoc comparisons within each treatment group (between time points) identified impaired reaching performance at post-stroke day 7 that improved by post-stroke day 42 and $56(p<0.05)$. However, only the ChABC group showed significant improvement after spinal injections, with improved performance at post-stroke day $56\left({ }^{\times} p<0.05\right)$ relative to day 28. C, There was no effect of time or treatment in sham-stroke controls $(p>0.05)$. D, There was no significant effect of treatment on spontaneous forelimb use measured in the cylinder task ( $p>0.05$ ); however, there was a significant main effect of time $(p<0.0001)$. There were also no significant differences in performance between 28 and $56 \mathrm{~d}$ in either group. $\boldsymbol{E}$, There was no effect of time or treatment in sham-stroke controls $(p>0.05)$. $\boldsymbol{F}$, A significant effect of time and interaction was detected in measures of mechanical sensitivity (time: $p<0.0001$; interaction: $p=0.0249$ ), but there were no significant differences between treatment groups at any time point after stroke. $\mathbf{G}$, There was no effect of time or treatment on mechanical sensitivity in sham-stroke animals.

with control-treated animals. This greater distribution of CST fibers throughout the cervical gray matter in ChABC-treated rats is apparent in camera lucida diagrams (Fig. $4 H, I$ ). Fiber-density heat plots illustrating the mean distribution of axon fragments confirmed that $\mathrm{ChABC}$ treatment promoted axonal sprouting throughout the cervical spinal gray matter with extensive innervation of lamina VII and lamina VI nearest to the CST (Fig. $4 J, K)$. Importantly, these effects could not be explained by dif- ferences in infarct volume, location, or differences in tracer injections between treatment groups (Fig. $2 A-F$ ).

ChABC potentiates task-specific rehabilitative training during chronic stroke

ChABC injection induced significant CST plasticity but only a moderate (in comparison) sensorimotor improvement. Exposing rats to rehabilitative training early after injury can reduce 
A

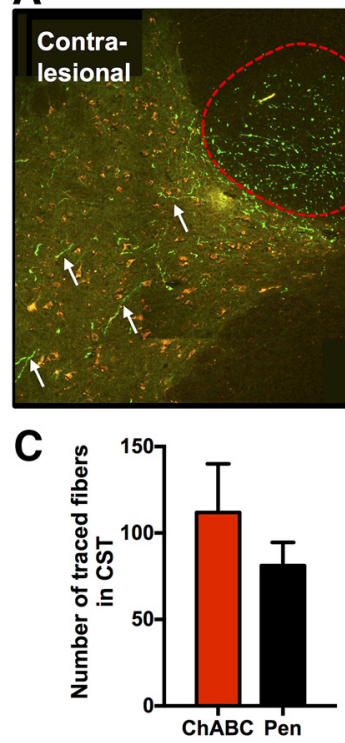

F
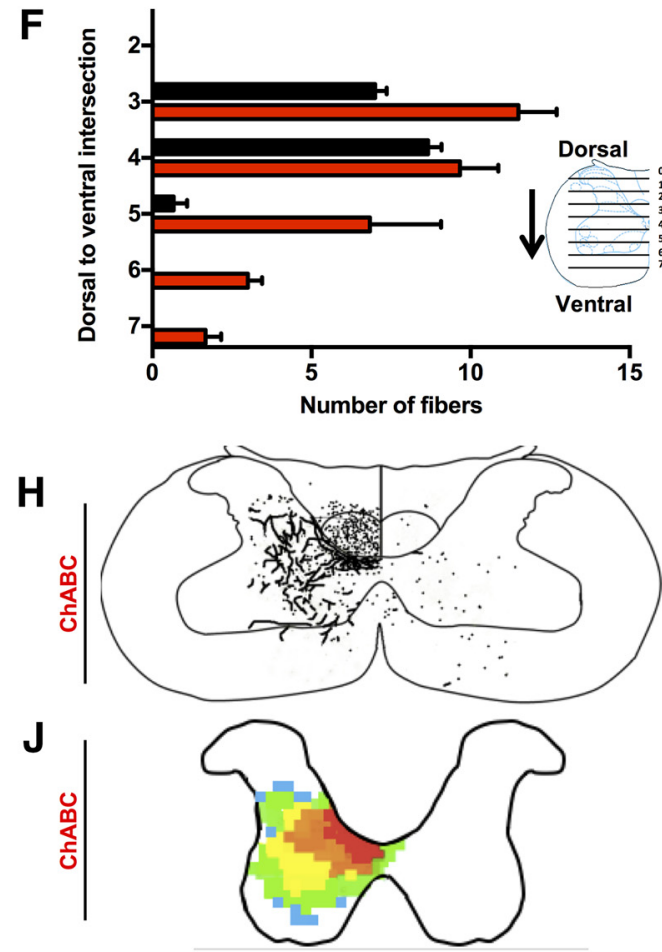

ChABC

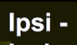

lesional

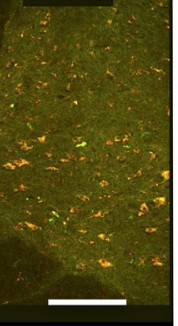

D

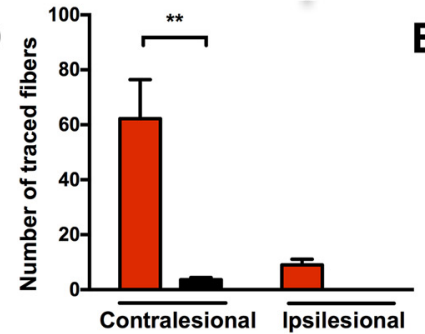

B

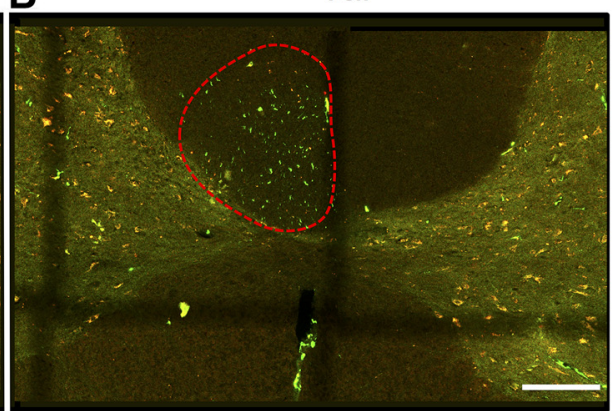

E

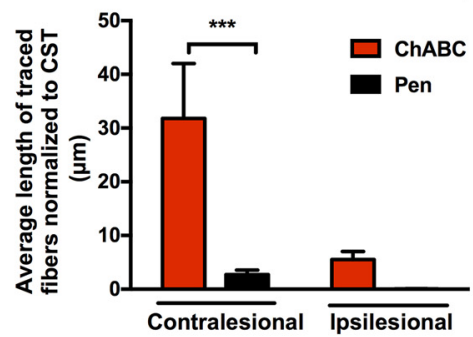

G
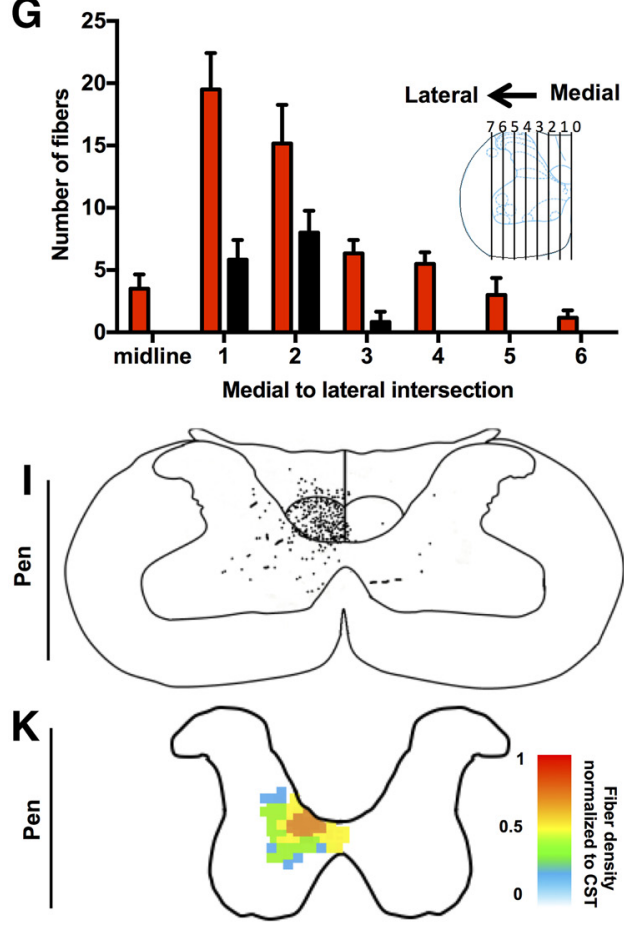

Figure 4. Delayed ChABC induces axonal sprouting of stroke deinnervated axons. $A, B$, Confocal microscopy of axons labeled with anterograde AlexaFluor 488-tagged dextran tracers (visualized using spectral settings for the FITC emission spectrum on the confocal microscope) are shown in in green. Images were also collected using a Rhodamine filter set to confirm that fluorescence from axon fragments were specific to the tracer (10X). Scale bars, $2 \mathrm{~mm}$. $\boldsymbol{A}$, terminals (arrows) in the contralesional (stroke-denervated) CST (outlined in red) and the contralesional and ipsilesional spinal cord gray matter in transverse sections spanning $C 4$ and $C 5$ of the cervical spinal cord of ChABC-treated rat. B, A transverse section through C 4 in a Pen control rat. Note the reduced density of labeled axon fragments from the CST in the gray matter relative to the ChABC-treated animal in $A$. C, Following stroke there was no significant difference in the number of labeled fibers in the CST between treatment groups $(p=0.38$; two-tailed, $t=0.91, \mathrm{df}=16)$. D. A significant main effect of treatment on the number of labeled axon fragments in the cervical gray matter was detected $(p=$ 0.0002). Significantly more fibers were found in the spinal gray matter contralateral to the cortical infarct of ChABC-treated animals $(* * * p<0.001) . \boldsymbol{E}$, There was a significant effect treatment $(p=$ 0.0034) on the average length of axon fragments in the spinal gray matter (normalized within animals to the total number of labeled fibers in the CST), with post hoc comparisons confirming greater axon length in the contralateral spinal gray matter of ChABC-treated animals $\left.{ }^{* *} p<0.01\right) . F$, ChABC increases dorsal to ventral and $(\boldsymbol{G})$ medial to lateral distribution of fibers in the gray matter of the contralesional cervical spinal cord. $\boldsymbol{H}-\boldsymbol{K}$, Camera lucida overlay tracings $(\boldsymbol{H}, \boldsymbol{I})$ and density heat maps $(\boldsymbol{J}, \boldsymbol{K})$ showing considerably increased density and distribution of axon fragments after $\mathrm{ChABC}$ treatment relative to Pen controls.

functional deficits but becomes ineffective during chronic stroke, potentially due to a closure of the critical period for heightened plasticity that follows in the weeks after stroke. Our anatomical data suggest that $\mathrm{ChABC}$ can recreate a window of heightened spinal plasticity in chronic stroke, which could in turn potentiate task-specific training. To test this, rats were trained on the single pellet reaching task before stroke and tested 3, 7, 14, 21, 28, 35, 42, 49 , and $56 \mathrm{~d}$ post-stroke (Fig. $5 \mathrm{~A}$ ). One day after injection with $\mathrm{ChABC}$ or Pen control (28 d post-stroke), rats were divided into groups that received either moderate task-specific rehabilitative 
A

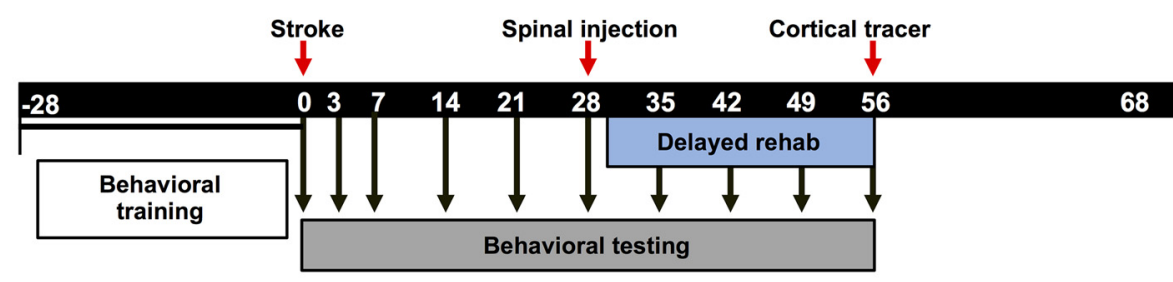

B

Stroke + delayed moderate rehab

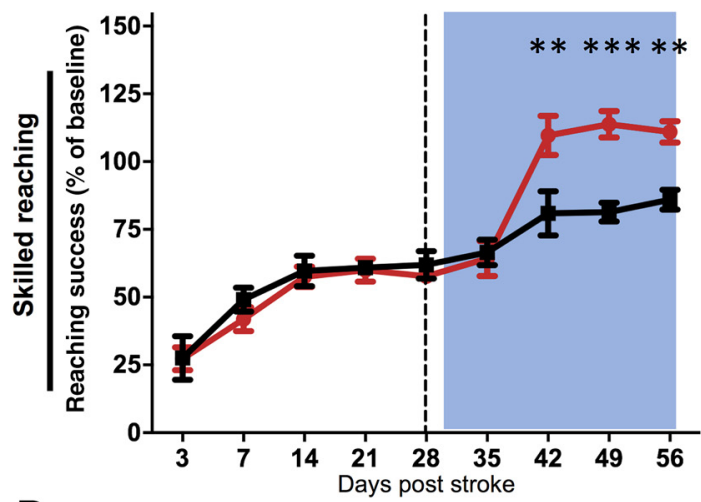

D

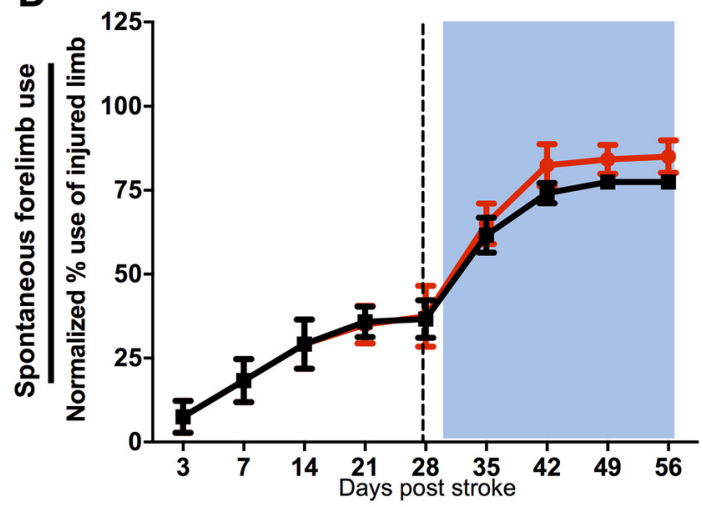

$\mathbf{F}$

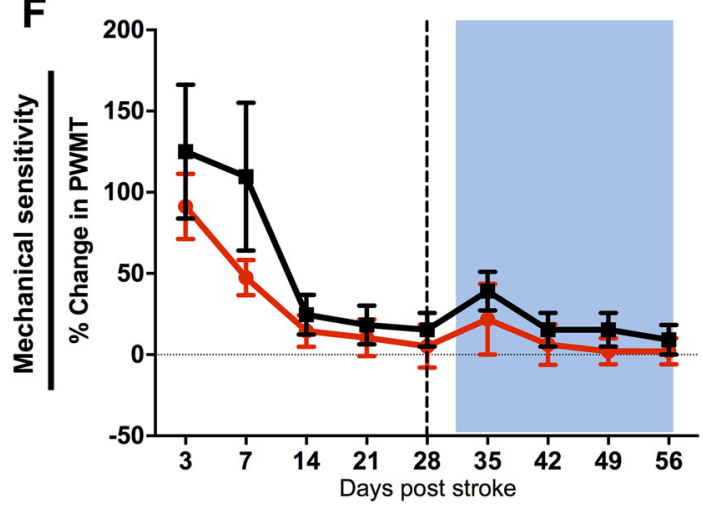

Stroke + delayed high intensity rehab
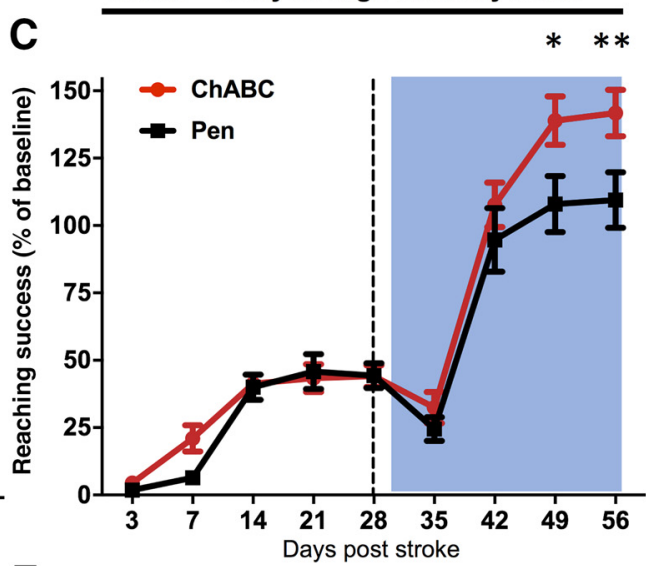

E

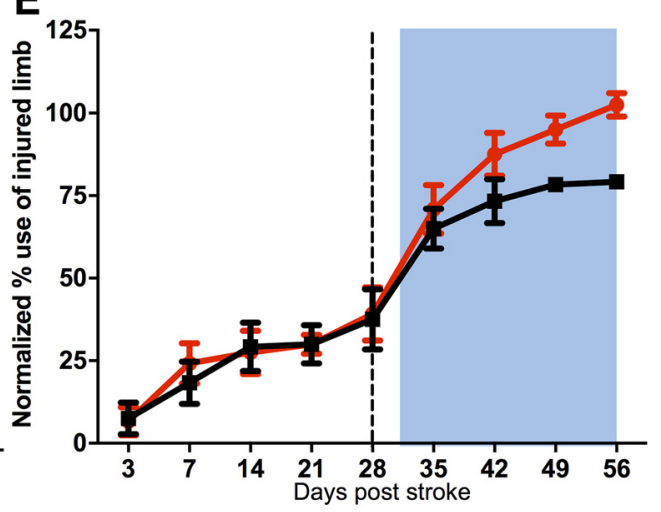

G

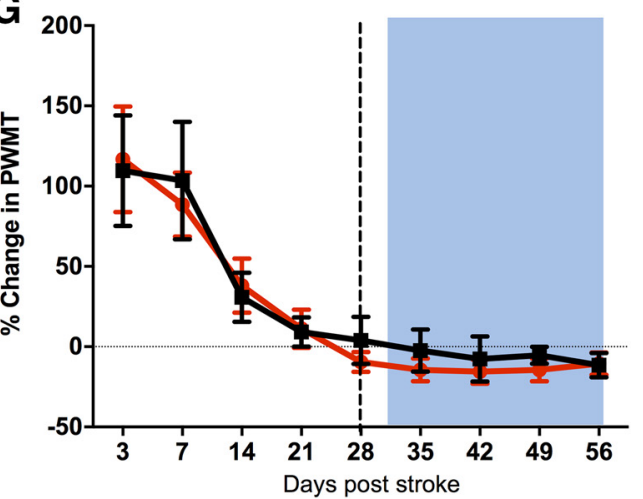

Figure 5. ChABC with delayed rehabilitative training of moderate or high intensity improves functional outcome. $A$, Timeline showing delayed rehabilitative training starting $28 \mathrm{~d}$ postinjury. $B$, Skilled reaching performance in the single pellet reaching task. There was a significant effect of time $(p<0.0001)$ and a significant time $\times$ treatment interaction $(p<0.0001)$. Holm-Sidak's multiple comparisons were used to compare the reaching performance of $\mathrm{ChABC}$-treated animals with Pen controls receiving the same training at each time point. ChABC-treated animals performed significantly better than the Pen controls after treatment at $42\left({ }^{* *} p<0.01\right), 49\left({ }^{* * *} p<0.001\right)$, and $56\left({ }^{* *} p<0.01\right)$ days post-stroke. There were no differences at time points before intraspinal injections. $C$, ChABC rats and Pen controls that received high-intensity training recovered to pre-stroke forelimb reaching performance. There was a significant interaction of time $\times$ treatment $\left(F_{(8,80)}=2.682, p=0.0115\right)$, as well as a significant effect of time $(p<0.0001)$. ChABC-treated animals performed significantly better than Pen controls at $49\left({ }^{*} p<0.05\right)$ and $56\left({ }^{* *} p<0.01\right)$ days after stroke. $\boldsymbol{D}, \boldsymbol{E}$, Animals receiving ChABC or Pen injections after injury combined with delayed rehabilitative training of moderate $(\boldsymbol{D})$ or high intensity $(\boldsymbol{E})$ exhibited no significant effect of treatment or interaction of time and treatment on the cylinder task to assess forelimb use preference. $\boldsymbol{F}$, $\boldsymbol{G}$, Similarly, mechanical sensitivity was unaffected by ChABC or Pen treatment. 
training (100 reaching attempts twice per day in the single pellet reaching apparatus) or high-intensity training (1000 reaching attempts twice per day) on days 29-56 post-stroke. These paradigms were chosen to model moderate and intense rehabilitative training and determine whether ChABC could make limited training more effective and/or remove plateaus that occur with intense training. Multivariate analyses revealed a significant interaction of time and treatment on reaching performance for both delayed moderate training $\left(F_{(8,80)}=7.344, p<0.0001\right.$; Fig. $5 B)$ and delayed high-intensity training $\left(F_{(8,80)}=2.682, p=\right.$ 0.0115; Fig. $5 C$ ). In moderate training conditions, ChABCtreated rats exhibited significantly better reaching success (relative to controls) at 42 (Holm-Sidak, $p<0.01), 49(p<0.001)$, and $56(p<0.01)$ days post-stroke. Notably, controls had only limited improvement from delayed moderate training, whereas ChABC-treated rats showed a dramatic improvement in testing on day 42. This delay between $\mathrm{ChABC}$ injection and sensorimotor improvement is consistent with ChABC enzyme kinetics and with previous work in various models of CNS injuries showing that the timing of spinal therapy relative to rehabilitative training is crucial (García-Alías et al., 2009; Soleman et al., 2012; Wahl et al., 2014). Both injection groups showed significant improvement in reaching performance during the high-intensity training. However, ChABC-injected rats exhibited superior performance at $49(p<0.05)$ and $56 \mathrm{~d}(p<0.01)$ after stroke relative to controls. Surprisingly, reaching performance at $56 \mathrm{~d}$ post-stroke was improved relative to baseline performance in ChABC-treated rats $(p<0.05)$. In both the moderate and high-intensity training groups, ChABC did not alter performance on the cylinder or induce any changes to spontaneous forelimb use preference (Fig. $5 D, E)$, nor did ChABC treatment induce aberrant mechanical sensitivity in von Frey test scoring (Fig. $5 F, G$ ). Although a main effect of time was observed in both assays (cylinder task: $F_{(1,80)}=$ 85.57, $p<0.0001$; von Frey test: $F_{(1,80)}=207.5, p<0.001$ ), there were no main effects of treatment or significant interactions or significant Holm-Sidak comparisons between time points after treatment.

Effects of delayed ChABC with early rehabilitative training The final experimental paradigm was designed to model permanent disability due to stroke even after extensive rehabilitation. Ideally, rehabilitative motor training is initiated early after stroke, as an early onset has been shown to exceed the benefits of delayed therapy in restoring behavioral performance in human clinical studies (Ottenbacher and Jannell, 1993; Paolucci et al., 2000; Musicco et al., 2003; Langhorne et al., 2010) and preclinical animal studies (Risedal et al., 1999; Farrell et al., 2001; Biernaskie et al., 2004; for review, see Teasell et al., 2005; Murphy and Corbett, 2009; Krakauer et al., 2012; Tennant, 2014). Rehabilitative training is continued into chronic stroke, though it loses efficacy with time and most patients remain disabled. To model this scenario, we delivered moderate task-specific training starting $3 \mathrm{~d}$ poststroke and continuing for $56 \mathrm{~d}$ (Fig. 6A). Early rehabilitative training induced marked improvement in skilled reaching and forelimb asymmetry in all animals by $28 \mathrm{~d}$ post-stroke (from $3.4 \pm 0.5 \%$ of baseline post-stroke to $74.2 \pm 4.5 \%$ of baseline in controls before treatment and from $4.4 \pm 0.5 \%$ to $65.2 \pm 4.5 \%$ of baseline in ChABC groups before spinal injections). However, control animals showed minimal improvement in reaching score (16.8 $\pm 5.0 \%$ improvement) with additional training between 28 and $56 \mathrm{~d}$ post-stroke, whereas ChABC injection on day 28 significantly potentiated the efficacy of rehabilitative training after treatment $(52.4 \pm 4.2 \%$ improvement). Multivariate ANOVA
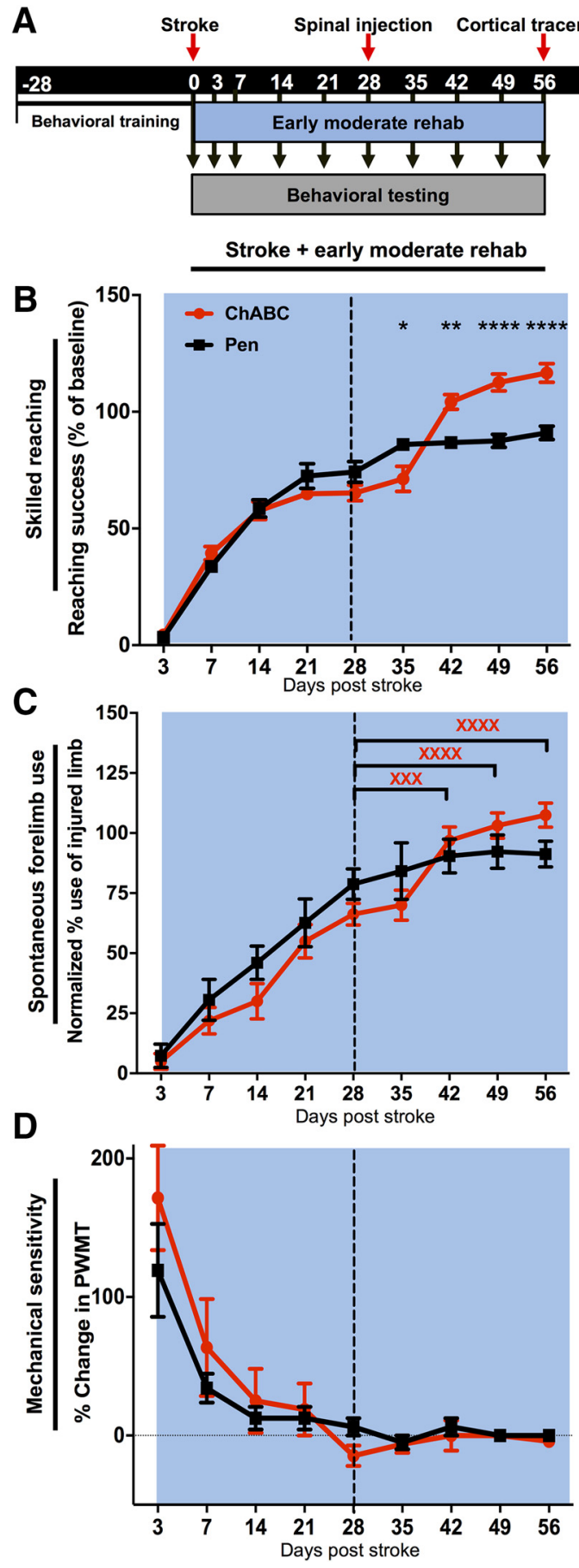

Figure 6. ChABC paired with early moderate rehabilitative training induced significant recovery of sensorimotor function. $A$, Timeline of testing, surgical procedures, and training beginning $3 \mathrm{~d}$ post-stroke. $\boldsymbol{B}$, There was a significant effect of time $(p<0.0001)$ and a significant interaction of treatment $\times$ time $(p<0.0001)$ in rats treated with moderate rehabilitative training early after stroke. Notably, $\mathrm{ChABC}$-treated animals had significantly better reaching performance than Pen controls at $42\left({ }^{* *} p<0.01\right), 49\left(^{* * * *} p<0.00001\right)$, and $56\left({ }^{* * * *} p<\right.$ 0.00001 ) days post-stroke (despite the fact that $\mathrm{ChABC}$ animals actually performed significantly worse than Pen-treated animals at $35 \mathrm{~d}$ post-stroke ( ${ }^{*} p<0.05$ ). C, There was no significant effect of treatment in the cylinder task testing; however, there was a significant effect of time $(p<0.0001)$. Within group post hoc comparisons revealed that only the ChABC-treated animals significantly increased use of their affected limb on days 42 ( $\left.{ }^{x x x} p<0.001\right), 49$ ( ${ }^{x x x x} p<$ 0.0001 ), and 56 ( $\left.{ }^{\mathrm{xxx}} p<0.0001\right)$ post-stroke compared with day 28 limb use preference. $D$, There were no treatment effects or significant interactions on assays of mechanical sensitivity. 

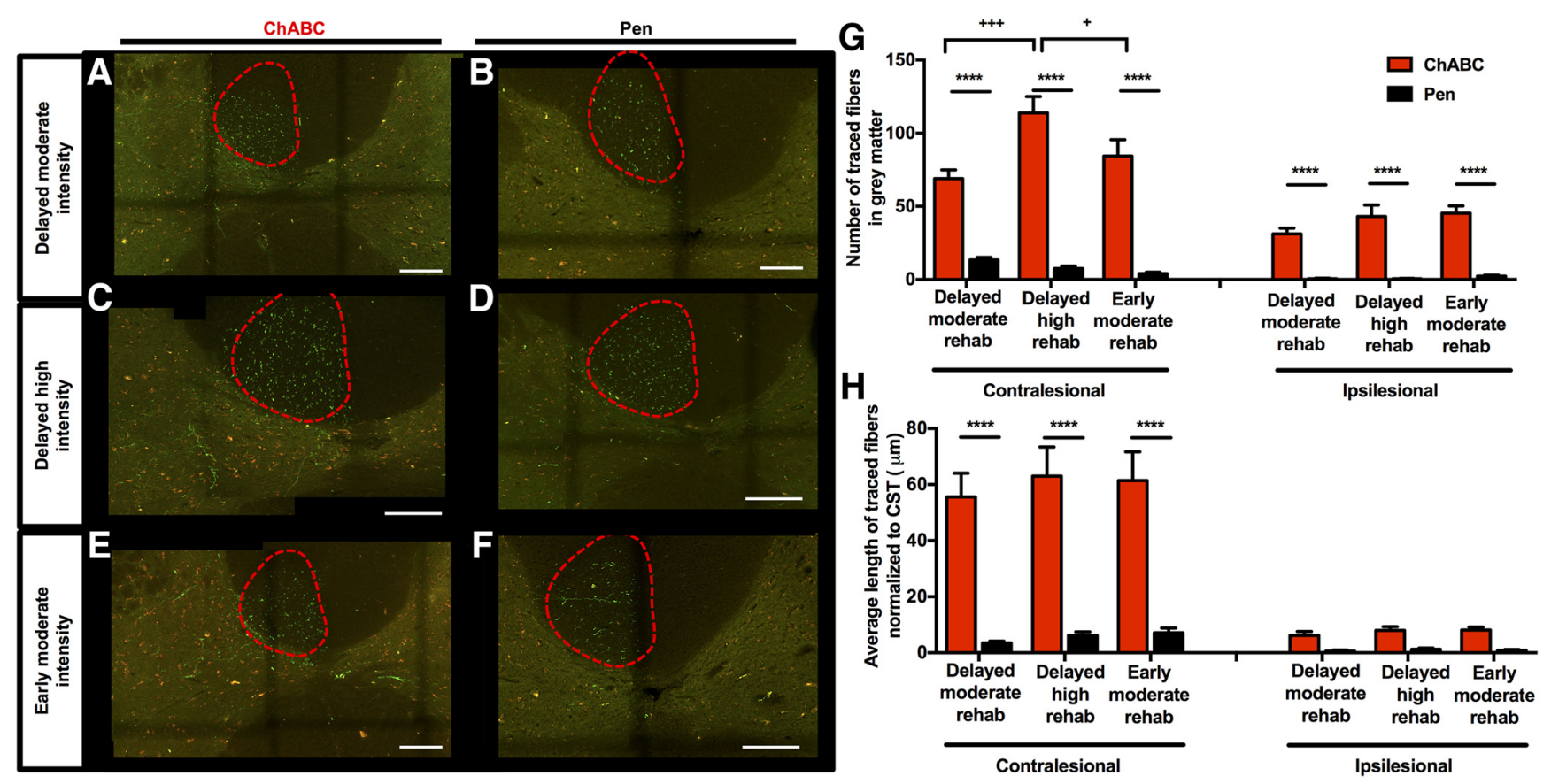

Figure 7. Histological analysis of anterograde tracer in animals receiving rehabilitative training. $\boldsymbol{A}-\boldsymbol{F}$, Confocal microscopy of transverse sections through C 4 showing CST fibers originating from injections in the peri-infarct cortex labeled with Anterograde AlexaFluor 488-tagged dextran tracers (visualized using spectral settings for the FITC emission spectrum on the confocal microscope) in green. Images were also collected using a Rhodamine filter set to confirm that fluorescence from axon fragments were specific to the tracer, in red. Representative sections are shown for animals receiving ChABC or Pen injection in the different rehabilitative training groups. Scale bars, $2 \mathrm{~mm}$. G, Gray matter fiber counts showed a significant effect of treatment in both contralesional ( $p<$ $0.0001)$ and ipsilesional $(p<0.0001)$ gray matter. Significantly greater numbers of axon fragments were found in all ChABC-treated groups relative to Pen controls receiving the same training $\left({ }^{* * * *} p<0.0001\right)$. Notably, there was also a significant main effect of training on the contralesional side $(p=0.0153)$ and a significant interaction $(p=0.027)$, with delayed high-intensity training significantly increased the number of labeled axon fragments in the gray matter in ChABC-treated animals relative to ChABC-treated animals receiving delayed $\left({ }^{+++} p<0.001\right)$ or early $\left({ }^{+} p<\right.$ $0.05)$ training at a moderate intensity. $\boldsymbol{H}$, A significant main effect of treatment $(p<0.0001)$ was found on measures of average axon length in the spinal gray matter, with significantly greater axon length in ChABC-treated animals $\left({ }^{* * *} p<0.0001\right)$ relative to Pen controls in every training group. There was no main effect of training group or significant interaction.

identified a significant interaction between treatment and time $\left(F_{(8,112)}=10.84, p<0.0001\right.$; Fig. $\left.6 B\right)$, and Holm-Sidak's multiple comparisons revealed that ChABC-treated animals had significantly better reaching performance than control animals at 42 $(p<0.01), 49(p<0.00001)$, and $56(p<0.00001)$ days poststroke (despite an initial impairment relative to controls at $35 \mathrm{~d}$ post-stroke $(p<0.05$; Fig. $6 B)$. There was no significant effect of treatment on cylinder reaching forelimb use scores; however, post hoc comparisons based on a significant main effect of time $\left(F_{(8,126)}=48.128, p<0.0001\right)$ suggest that ChABC-treated rats used their stroke affected limb significantly more on days 42 ( $p<$ $0.001), 49(p<0.001)$, and $56(p<0.0001)$ than on day 28 (pre-treatment). The same effect was not observed in controls (Fig. $6 C$ ). As in the case of the delayed training groups, there was a significant effect of time $\left(F_{(8,112)}=19.71, p<0.0001\right)$ but no effect of ChABC injection on mechanical sensitivity or significant comparisons between time points after treatment (Fig. 6D).

\section{Effects of combined therapy on CST and serotonergic innervation of the cervical cord}

Analysis of anterograde tracers injected into peri-infarct motor cortex shows that rats that received training paired with ChABC exhibited significantly higher fiber counts (contralesional gray matter: $F_{(1,54)}=201.0, p<0.0001$; ipsilesional: $F_{(1,54)}=130.3$, $p<0.0001$; Fig. $7 G)$ and significantly greater fiber lengths $\left(F_{(1,54)}=\right.$ $91.50, p<0.0001$; Fig. $7 H$ ) than rats that received training paired with Pen injection. High intensity training resulted in a greater number (but not length) of fibers on the contralesional side than the early $(p<0.05)$ or delayed moderate $(p<0.001)$ training groups (Fig. $7 G, H$ ). Notably, ChABC-treated animals in the early rehabilitative training group exhibited a 20 -fold increase in the number of fibers relative to controls, while animals in the delayed moderate and delayed high-intensity training groups exhibited fivefold and 16-fold increases in fiber number relative to controls. Importantly, there was no difference in infarct volume or locations of anterograde tracer injections between treatment groups in any of the studies involving rehabilitative training (Fig. $2 A-F$ ).

ChABC induced significant sprouting of CST derived axons in the spinal cord. Although nociceptive fibers in the dorsal spinal cord were not assessed, the lack of behavioral effects on tests of pain sensitivity suggests that significant sprouting did not occur. To further assess whether structural plasticity induced by ChABC was specific to the CST, the density of serotonergic axons projecting to the gray matter of the cervical spinal cord at the $\mathrm{C} 4$ level was also assessed. Moreover, because the serotonergic system has been reported to be involved in compensatory mechanisms and recovery that follows spinal injury, and because serotonin has a crucial role in regulating the excitability of spinal motor neurons (Hounsgaard et al., 1988), plasticity of the serotonergic projections may contribute to recovery. A significant main effect of treatment paradigm on serotonergic fiber density in the contralesional spinal cord was observed (ANOVA, $F_{(8,36)}=7.428, p<$ $0.0001)$. Serotonergic fiber density was reduced by stroke ( $p<$ 0.05 , relative to sham-stroke controls), an effect that was not reversed by ChABC alone ( $p>0.05$, relative to shams). However, when $\mathrm{ChABC}$ was paired with moderate or intense delayed rehabilitative training (Fig. $8 A, C$ ), serotonergic innervation was not significantly different from sham-stroke controls. Pen controls still exhibited significantly reduced serotonergic fiber density relative to controls $(p<0.01)$. Notably, early rehabilitative training 
A
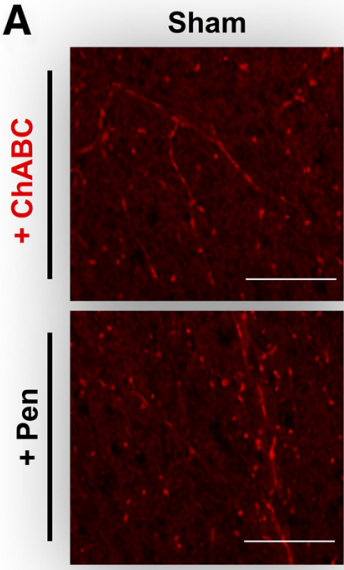

B

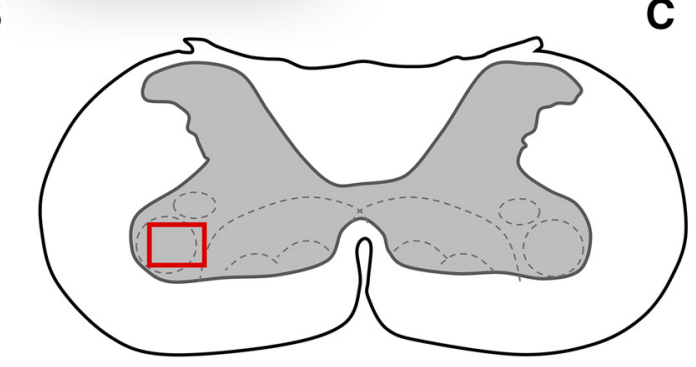

No Rehab
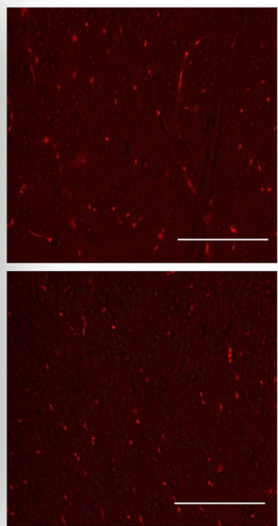

C
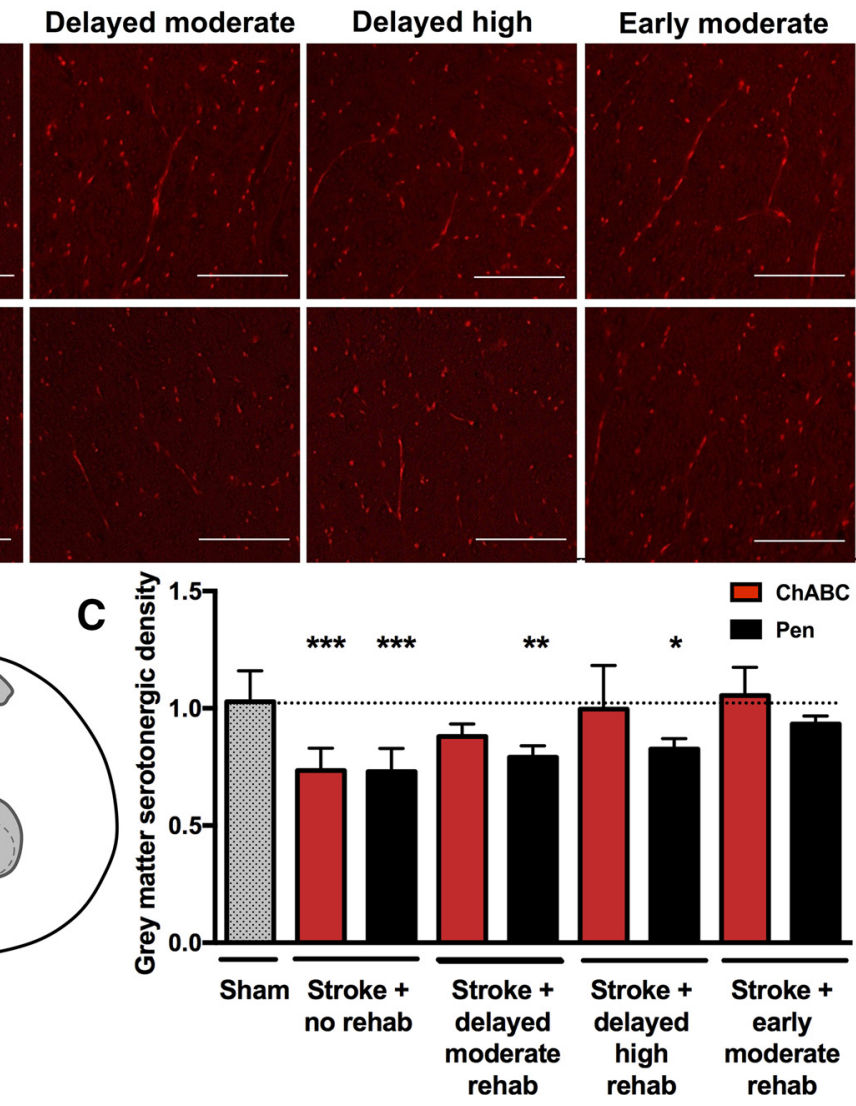

Figure 8. Changes in serotonergic fiber distribution in the cervical spinal cord (C4) gray matter. $\boldsymbol{A}$, Serotonergic fibers from the area of the ventral horn delineated in $\boldsymbol{B}$ from ChABC-treated rats or Pen controls under different training paradigms. Serotonergic fibers are labeled with anti-serotonin antibody and Texas red secondary antibody (visualized using spectral settings for the Cy 3 emission spectrum on the confocal microscope) at $10 \times$ magnification. Scale bar, $100 \mu \mathrm{m}$. $\boldsymbol{B}$, Schematic diagram of the (4/C5 transverse spinal cord. Red box delineates area of ventral horn gray matter selected for optical density quantification. C, There was a significant main effect of treatment group on serotonergic density in the contralesional spinal gray matter (ANOVA, $p<0.0001$ ). Notably, stroke significantly reduced contralesional serotonergic density relative to sham animals, and this effect was not altered by ChABC treatment alone (without rehabilitative training; ${ }^{* * *} p<$ 0.001). However, in ChABC-treated animals receiving delayed training (both moderate and high intensity), there was no statically significant difference relative to shams. Pen controls receiving delayed training exhibited significantly reduced serotonergic density $\left({ }^{* *} p<0.01,{ }^{*} p<0.05\right)$. Early rehabilitative training recovered serotonergic density, with no significant difference between ChABC-treated rats and Pen controls.

restored serotonergic axon density in controls and ChABCtreated rats, though a trend toward synergistic effect remained.

\section{Discussion}

Almost all recovery after stroke occurs in the first few weeks after injury. Even with extensive and sustained rehabilitative training, sensorimotor recovery peaks by 4 weeks in rodents (Biernaskie et al., 2004; Hsu and Jones, 2006; Alaverdashvili et al., 2008) and 3 months in humans (Andrews et al., 1981; Skilbeck et al., 1983; Wade et al., 1983; Smith et al., 1985; Duncan et al., 1992; Nakayama et al., 1994; Lo et al., 2010; for reviews, see Steinberg and Augustine, 1997; Teasell et al., 2005; Krakauer et al., 2012; Wahl and Schwab, 2014). Moreover, the contribution of rehabilitative motor training to this recovery has been questioned. Neutral or negative results in randomized clinical trials have led to questions about the efficacy with which rehabilitative training can improve function above spontaneous biological recovery (Lowry, 2010; AVERT Trial Collaboration Group, 2015; Krakauer and Marshall, 2015; Winstein et al., 2016). In this project, we investigated whether ChABC therapy delivered to the spinal cord during the chronic phase of stroke recovery could improve recovery and augment rehabilitation even after the optimal window for recovery that follows early after stroke. Despite notable increases in the number, length, and distribution of CST axons originating in peri-infarct cortex and projecting to the cervical gray matter, there was only limited functional benefit of ChABC alone (without rehabilitative training). This is not entirely surprising as previous studies in $\mathrm{C} 4$ spinal cord injury models indicated that ChABC only allowed for improvement in sensorimotor function when combined with rehabilitation (García-Alías et al., 2009). Similar findings have been reported with other plasticity promoting treatments such as intracortical brain-derived neurotrophic factor (Vavrek et al., 2006; Weishaupt et al., 2013). To determine whether this enhanced plasticity could be harnessed to rekindle the effectiveness of task-specific training, we paired ChABC with delayed reaching training in the single pellet task. Delayed rehabilitative training of moderate intensity was ineffective when paired with control injection, but ChABC paired with training induced significant improvement. Rats that underwent intense reaching training starting after $\mathrm{ChABC}$ injection on day 28 showed significant improvement, with the ChABC-treated animals again performing significantly better than controls. To model stroke patients who receive early rehabilitation that is continued through to chronic stroke, but whose recovery plateaus in the weeks following the initial injury, another cohort of rats received moderate rehabilitative training starting $3 \mathrm{~d}$ after stroke and continuing for 8 weeks. Notably, whereas rehabilitative 
training efficacy plateaued by $28 \mathrm{~d}$ after stroke and was not significantly improved by control injection, rats exhibited amplified training-induced recovery after intraspinal $\mathrm{ChABC}$ at $28 \mathrm{~d}$. These data suggest that intraspinal $\mathrm{ChABC}$ can significantly improve training induced recovery in a model approximating chronic stroke patients. Consistent with previous preclinical (Wang et al., 2016; El Amki et al., 2017) and clinical studies (Sunderland et al., 1992; Kwakkel et al., 1997, 1999; Lang et al., 2009), our paradigm of extremely intense rehabilitative training (2000 reaches per day) was associated with the best sensorimotor improvement. More moderate rehabilitative training (200 reaches per day) was effective when initiated early after stroke (with a plateau in recovery after 2-3 weeks), but provided less benefit when initiated $28 \mathrm{~d}$ after stroke. In all groups, rehabilitative training was improved by intraspinal ChABC at $28 \mathrm{~d}$ after stroke, suggesting that optimal rehabilitation can be attained by combining pro-plasticity therapy with training.

Although our treatments were designed to "reopen" the window for plasticity, the efficacy of intense rehabilitative training in improving reaching performance during chronic stroke even without $\mathrm{ChABC}$ injection suggests that the window for plasticity was not completely ended by 4 weeks after stroke. Indeed, the magnitude of the gains due to rehabilitation (particularly intense training) was greater than the additional benefit of adding ChABC. However, our data suggest that ChABC can potentiate plasticity of the CST and improve delayed rehabilitative training. Spinal treatment improved the efficacy of a moderate level of rehabilitation and also amplified the efficacy of rehabilitative training performed at maximum intensities (overcoming potential ceiling effects of intense training). Given that the efficacy of training after stroke has been questioned in light of negative results in clinical trials, a treatment that can make moderate or intense rehabilitation paradigms more effective is promising. Studies using an even longer recovery period would be needed to conclusively demonstrate a "reopening" of the critical period for recovery. The efficacy of intense rehabilitation administered during chronic stroke has been reported in stroke patients undergoing intense training. For example, clinical investigations of constrained induced movement therapy starting 3-9 months after stroke that demonstrated that intense training can induce improvement in arm function for stroke patients even during chronic stroke (Wolf et al., 2006; McIntyre et al., 2012; Lang et al., 2013). Similarly, preclinical studies of chronic spinal cord injury have shown that ChABC can improve outcome (García-Alías et al., 2009) and the effects ChABC administered at time points early after spinal injury are amplified with training (García-Alías et al., 2009).

Intraspinal ChABC exhibited a pro-plasticity effect on CST axons originating in peri-infarct cortex. Several studies have shown that plasticity enhancing treatments increase sprouting of the uninjured CST into the stroke-deinnervated hemicord and improve recovery from cortical injury, and that blocking the sprouting of these fibers impairs sensorimotor recovery (Soleman et al., 2012; Wahl et al., 2014). However, although the plasticity of the uninjured CST has been elegantly demonstrated to contribute to recovery in animal models (stroke: Soleman et al., 2012; Wahl et al., 2014; traumatic brain injury model: Ueno et al., 2012; spinal injury: Cafferty and Strittmatter, 2006; Starkey et al., 2012; CST brainstem lesions: Thallmair et al., 1998), clinical studies of cortical reorganization after stroke suggest that better recovery is associated with reorganization close to the site of injury (Johansen-Berg et al., 2002a,b; Fridman et al., 2004) as opposed to reorganization in distal regions such as the contralateral cortex (Calautti and Baron, 2003; Schaechter, 2004). Thus, we were interested in whether significant sprouting of spared peri-infarct CST projections would occur with spinal therapy. Our data confirms plasticity of the perilesional CST, though the relative contributions of uninjured and spared CST to post-stroke recovery, as well as contributions from plasticity in local spinal connections, remain to be investigated.

By inducing sprouting of nociceptive fibers, intraspinal ChABC could potentiate mechanical sensitivity and induce hyperalgesia. However, von Frey hair testing did not indicate the development of pathological pain circuitry, as hyperalgesia (reduced PWMTs relative to baseline) was not observed in any treatment groups. Our data did suggest an interaction between $\mathrm{ChABC}$ treatment, rehabilitative training, and the density of serotonergic innervation of the cervical spinal cord. Whereas ChABC alone did not induce an increase in serotonergic fiber density, ChABC paired with delayed training significantly increased serotonergic innervation. Following spinal cord injury, serotonergic input is lost to regions of the spinal cord distal to the site of injury (Fong et al., 2005). Interestingly, our data suggest that cortical stroke also significantly reduces serotonergic innervation of the spinal cord, despite the lack of overt injury to serotonergic fibers. Direct application of serotonin to deinnervated spinal cord sites restores spinal excitability and can improve motor function (Fong et al., 2005), indicating that restoration of serotonergic innervation is important for motor recovery after injury (Leszczyńska et al., 2015). Removal of growth inhibitory factors in the glial scar potentiates regrowth of serotonergic fibers and improves recovery after spinal cord injury (Camand et al., 2004; Müllner et al., 2008). Although spontaneous regrowth of serotonergic fibers after spinal injury occurs over the first 4 weeks after spinal injury (Leszczyńska et al., 2015), there is evidence that suggests serotonergic neurons have late intrinsic growth programs and continue to sprout over long periods (1-6 months) after injury (Li and Raisman, 1995; Hill et al., 2001; Filli et al., 2011). Increased serotonergic fiber density is one postulated mechanism of improved sensory motor recovery in $\mathrm{ChABC}$-treated and rehabilitated animals after spinal injury (Jacobs et al., 2002). Moreover, increases in the length of serotonergic fibers after injury correlate with improvement in both inter- and intra-limb coordination (Leszczyńska et al., 2015), which are crucial in skilled reaching tasks.

In summary, our data show that removal of growth inhibitory CSPGs in the spinal cord of rats during chronic stroke can augment structural plasticity in the spared CST, amplify the functional benefit of delayed rehabilitative training, and induce improved training efficacy after the period for optimal rehabilitation efficacy. These data therefore suggest that the permanent disability affecting millions of individuals living with the chronic effects of stroke may be treatable with spinal therapy and rehabilitation initiated even months or years after the stroke. Our data also emphasize that inducing a state of plasticity is not sufficient to induce recovery, and that combining such therapies with rehabilitative training is required for optimal recovery (Girgis et al., 2007).

\section{References}

Afshari FT, Kwok JC, White L, Fawcett JW (2010) Schwann cell migration is integrin-dependent and inhibited by astrocyte-produced aggrecan. Glia 58:857-869. CrossRef Medline

Alaverdashvili M, Moon SK, Beckman CD, Virag A, Whishaw IQ (2008) Acute but not chronic differences in skilled reaching for food following motor cortex devascularization vs. photothrombotic stroke in the rat. Neuroscience 157:297-308. CrossRef Medline

Andrews K, Brocklehurst JC, Richards B, Laycock PJ (1981) The rate of 
recovery from stroke- and its measurement. Int Rehabil Med 3:155-161. CrossRef Medline

AVERT Trial Collaboration Group (2015) Efficacy and safety of very early mobilisation within $24 \mathrm{~h}$ of stroke onset (AVERT): a randomised controlled trial. Lancet 386:46-55. CrossRef Medline

Biernaskie J, Chernenko G, Corbett D (2004) Efficacy of rehabilitative experience declines with time after focal ischemic brain injury. J Neurosci 24:1245-1254. CrossRef Medline

Bradman MJ, Ferrini F, Salio C, Merighi A (2015) Practical mechanical threshold estimation in rodents using von Frey hairs/Semmes-Weinstein monofilaments: towards a rational method. J Neurosci Methods 255:92103. CrossRef Medline

Burnside ER, Bradbury EJ (2014) Review: manipulating the extracellular matrix and its role in brain and spinal cord plasticity and repair. Neuropathol Appl Neurobiol 40:26-59. CrossRef Medline

Cafferty WB, Strittmatter SM (2006) The nogo-nogo receptor pathway limits a spectrum of adult CNS axonal growth. J Neurosci 26:12242-12250. CrossRef Medline

Calautti C, Baron JC (2003) Functional neuroimaging studies of motor recovery after stroke in adults: a review. Stroke 34:1553-1566. CrossRef Medline

Camand E, Morel MP, Faissner A, Sotelo C, Dusart I (2004) Long-term changes in the molecular composition of the glial scar and progressive increase of serotoninergic fibre sprouting after hemisection of the mouse spinal cord. Eur J Neurosci. 20:1161-1176. CrossRef Medline

Cua RC, Lau LW, Keough MB, Midha R, Apte SS, Yong VW (2013) Overcoming neurite-inhibitory chondroitin sulfate proteoglycans in the astrocyte matrix. Glia 61:972-984. CrossRef Medline

Dimyan MA, Cohen LG (2011) Neuroplasticity in the context of motor rehabilitation after stroke. Nat Rev Neurol 7:76-85. CrossRef Medline

Dobkin BH (2008) Training and exercise to drive poststroke recovery. Nat Clin Pract Neurol 4:76-85. CrossRef Medline

Duncan PW, Goldstein LB, Matchar D, Divine GW, Feussner J (1992) Measurement of motor recovery after stroke. outcome assessment and sample size requirements. Stroke 23:1084-1089. CrossRef Medline

El Amki M, Baumgartner P, Bracko O, Luft AR, Wegener S (2017) Taskspecific motor rehabilitation therapy after stroke improves performance in a different motor task: translational evidence. Transl Stroke Res 8:347350. CrossRef Medline

Farrell R, Evans S, Corbett D (2001) Environmental enrichment enhances recovery of function but exacerbates ischemic cell death. Neuroscience 107:585-592. CrossRef Medline

Filli L, Zörner B, Weinmann O, Schwab ME (2011) Motor deficits and recovery in rats with unilateral spinal cord hemisection mimic the BrownSéquard syndrome. Brain 134:2261-2273. CrossRef Medline

Fisher D, Xing B, Dill J, Li H, Hoang HH, Zhao Z, Yang XL, Bachoo R, Cannon S, Longo FM, Sheng M, Silver J, Li S (2011) Leukocyte common antigen-related phosphatase is a functional receptor for chondroitin sulfate proteoglycan axon growth inhibitors. J Neurosci 31:14051-14066. CrossRef Medline

Fong AJ, Cai LL, Otoshi CK, Reinkensmeyer DJ, Burdick JW, Roy RR, Edgerton VR (2005) Spinal cord-transected mice learn to step in response to quipazine treatment and robotic training. J Neurosci 25:11738-11747. CrossRef Medline

Fridman EA, Hanakawa T, Chung M, Hummel F, Leiguarda RC, Cohen LG (2004) Reorganization of the human ipsilesional premotor cortex after stroke. Brain 127:747-758. CrossRef Medline

García-Alías G, Barkhuysen S, Buckle M, Fawcett JW (2009) Chondroitinase $\mathrm{ABC}$ treatment opens a window of opportunity for task-specific rehabilitation. Nat Neurosci 12:1145-1151. CrossRef Medline

Girgis J, Merrett D, Kirkland S, Metz GA, Verge V, Fouad K (2007) Reaching training in rats with spinal cord injury promotes plasticity and task specific recovery. Brain 130:2993-3003. CrossRef Medline

Green JB (2003) Brain reorganization after stroke. Top Stroke Rehabil 10:120. CrossRef Medline

Hara Y (2015) Brain plasticity and rehabilitation in stroke patients. J Nippon Med Sch 82:4-13. CrossRef Medline

Hill CE, Beattie MS, Bresnahan JC (2001) Degeneration and sprouting of identified descending supraspinal axons after contusive spinal cord injury in the rat. Exp Neurol 171:153-169. CrossRef Medline

Houle JD, Tom VJ, Mayes D, Wagoner G, Phillips N, Silver J (2006) Combining an autologous peripheral nervous system "bridge" and matrix modification by chondroitinase allows robust, functional regeneration beyond a hemisection lesion of the adult rat spinal cord. J Neurosci 26: 7405-7415. CrossRef Medline

Hounsgaard J, Hultborn H, Jespersen B, Kiehn O (1988) Bistability of alpha-motoneurones in the decerebrate cat and in the acute spinal cat after intravenous 5-hydroxytryptophan. J Physiol 405:345-367. CrossRef Medline

Hsu JE, Jones TA (2006) Contralesional neural plasticity and functional changes in the less-affected forelimb after large and small cortical infarcts in rats. Exp Neurol 201:479-494. CrossRef Medline

Jacobs BL, Martín-Cora FJ, Fornal CA (2002) Activity of medullary serotonergic neurons in freely moving animals. Brain Res Rev 40:45-52. CrossRef Medline

Johansen-Berg H, Dawes H, Guy C, Smith SM, Wade DT, Matthews PM (2002a) Correlation between motor improvements and altered fMRI activity after rehabilitative therapy. Brain 125:2731-2742. CrossRef Medline

Johansen-Berg H, Rushworth MF, Bogdanovic MD, Kischka U, Wimalaratna S, Matthews PM (2002b) The role of ipsilateral premotor cortex in hand movement after stroke. Proc Natl Acad Sci U S A 99:14518-14523. CrossRef Medline

Jones TA, Adkins DL (2015) Motor system reorganization after stroke: stimulating and training toward perfection. Physiology 30:358-370. CrossRef Medline

Kessner SS, Bingel U, Thomalla G (2016) Somatosensory deficits after stroke: a scoping review. Top Stroke Rehabil 23:136-146. CrossRef Medline

Kitago T, Krakauer JW (2013) Motor learning principles for neurorehabilitation. Handb Clin Neurol 110:93-103. CrossRef Medline

Krakauer JW, Marshall RS (2015) The proportional recovery rule for stroke revisited. Ann Neurol 78:845-847. CrossRef Medline

Krakauer JW, Carmichael ST, Corbett D, Wittenberg GF (2012) Getting neurorehabilitation right: what can be learned from animal models? Neurorehabil Neural Repair 26:923-931. CrossRef Medline

Kwakkel G, Wagenaar RC, Koelman TW, Lankhorst GJ, Koetsier JC (1997) Effects of intensity of rehabilitation after stroke: a research synthesis. Stroke 28:1550-1556. CrossRef Medline

Kwakkel G, Wagenaar RC, Twisk JW, Lankhorst GJ, Koetsier JC (1999) Intensity of leg and arm training after primary middle-cerebral-artery stroke: a randomised trial. Lancet 354:191-196. CrossRef Medline

Kwok JC, Dick G, Wang D, Fawcett JW (2011) Extracellular matrix and perineuronal nets in CNS repair. Dev Neurobiol 71:1073-1089. CrossRef Medline

Lang CE, MacDonald JR, Reisman DS, Boyd L, Jacobson Kimberley T, Schindler-Ivens SM, Hornby TG, Ross SA, Scheets PL (2009) Observation of amounts of movement practice provided during stroke rehabilitation. Arch Phys Med Rehabil 90:1692-1698. CrossRef Medline

Lang KC, Thompson PA, Wolf SL (2013) The EXCITE trial: reacquiring upper-extremity task performance with early versus late delivery of constraint therapy. Neurorehabil Neural Repair 27:654-663. CrossRef Medline

Langhorne P, Stott D, Knight A, Bernhardt J, Barer D, Watkins C (2010) Very early rehabilitation or intensive telemetry after stroke: a pilot randomised trial. Cerebrovasc Dis 29:352-360. CrossRef Medline

Leszczyńska AN, Majczyński H, Wilczyński GM, Sławińska U, Cabaj AM (2015) Thoracic hemisection in rats results in initial recovery followed by a late decrement in locomotor movements, with changes in coordination correlated with serotonergic innervation of the ventral horn. PLoS One 10:e0143602. CrossRef Medline

Li Y, Raisman G (1995) Sprouts from cut corticospinal axons persist in the presence of astrocytic scarring in long-term lesions of the adult rat spinal cord. Exp Neurol 134:102-111. CrossRef Medline

Lo AC, Guarino PD, Richards LG, Haselkorn JK, Wittenberg GF, Federman DG, Ringer RJ, Wagner TH, Krebs HI, Volpe BT, Bever CT Jr, Bravata DM, Duncan PW, Corn BH, Maffucci AD, Nadeau SE, Conroy SS, Powell JM, Huang GD, Peduzzi P (2010) Robot-assisted therapy for long-term upper-limb impairment after stroke. N Engl J Med 362:1772-1783. CrossRef Medline

Lowry F (2010) Stroke rehabilitation services inadequate, experts say. CMAJ 182:E283-E284. CrossRef Medline

Maier IC, Schwab ME (2006) Sprouting, regeneration and circuit formation in the injured spinal cord: factors and activity. Philos Trans R Soc Lond B Biol Sci 361:1611-1634. CrossRef Medline

McIntyre A, Viana R, Janzen S, Mehta S, Pereira S, Teasell R (2012) System- 
atic review and meta-analysis of constraint-induced movement therapy in the hemiparetic upper extremity more than six months post stroke. Top Stroke Rehabil 19:499-513. CrossRef Medline

Monnier PP, Sierra A, Schwab JM, Henke-Fahle S, Mueller BK (2003) The rho/ROCK pathway mediates neurite growth-inhibitory activity associated with the chondroitin sulfate proteoglycans of the CNS glial scar. Mol Cell Neurosci 22:319-330. CrossRef Medline

Moon LD, Asher RA, Rhodes KE, Fawcett JW (2001) Regeneration of CNS axons back to their target following treatment of adult rat brain with chondroitinase ABC. Nat Neurosci 4:465-466. CrossRef Medline

Müllner A, Gonzenbach RR, Weinmann O, Schnell L, Liebscher T, Schwab ME (2008) Lamina-specific restoration of serotonergic projections after nogo-A antibody treatment of spinal cord injury in rats. Eur J Neurosci 27:326-333. CrossRef Medline

Murphy TH, Corbett D (2009) Plasticity during stroke recovery: from synapse to behaviour. Nat Rev Neurosci 10:861-872. CrossRef Medline

Musicco M, Emberti L, Nappi G, Caltagirone C (2003) Early and long-term outcome of rehabilitation in stroke patients: the role of patient characteristics, time of initiation, and duration of interventions. Arch Phys Med Rehabil 84:551-558. CrossRef Medline

Nakayama H, Jørgensen HS, Raaschou HO, Olsen TS (1994) Recovery of upper extremity function in stroke patients: the copenhagen stroke study. Arch Phys Med Rehabil 75:394-398. CrossRef Medline

Ottenbacher KJ, Jannell S (1993) The results of clinical trials in stroke rehabilitation research. Arch Neurol 50:37-44. CrossRef Medline

Overman JJ, Carmichael ST (2014) Plasticity in the injured brain. Neuroscientist 20:15-28. CrossRef Medline

Paolucci S, Antonucci G, Grasso MG, Morelli D, Troisi E, Coiro P, Bragoni M (2000) Early versus delayed inpatient stroke rehabilitation: a matched comparison conducted in Italy. Arch Phys Med Rehabil 81:695-700. CrossRef Medline

Risedal A, Zeng J, Johansson BB (1999) Early training may exacerbate brain damage after focal brain ischemia in the rat. J Cereb Blood Flow Metab 19:997-1003. CrossRef Medline

Schaechter JD (2004) Motor rehabilitation and brain plasticity after hemiparetic stroke. Prog Neurobiol 73:61-72. CrossRef Medline

Sharma K, Selzer ME, Li S (2012) Scar-mediated inhibition and CSPG receptors in the CNS. Exp Neurol 237:370-378. CrossRef Medline

Shen Y, Tenney AP, Busch SA, Horn KP, Cuascut FX, Liu K, He Z, Silver J, Flanagan JG (2009) PTP $\sigma$ is a receptor for chondroitin sulfate proteoglycan, an inhibitor of neural regeneration. Science 326:592-596. CrossRef Medline

Silasi G, Murphy TH (2014) Removing the brakes on post-stroke plasticity drives recovery from the intact hemisphere and spinal cord. Brain 137: 648-650. CrossRef Medline

Silver J, Miller JH (2004) Regeneration beyond the glial scar. Nat Rev Neurosci 5:146-156. CrossRef Medline

Sist B, Fouad K, Winship IR (2014) Plasticity beyond peri-infarct cortex: spinal up regulation of structural plasticity, neurotrophins, and inflammatory cytokines during recovery from cortical stroke. Exp Neurol 252: 47-56. CrossRef Medline

Skilbeck CE, Wade DT, Hewer RL, Wood VA (1983) Recovery after stroke. J Neurol Neurosurg Psychiatry 46:5-8. CrossRef Medline

Smith DL, Akhtar AJ, Garraway WM (1985) Motor function after stroke. Age Ageing 14:46-48. CrossRef Medline

Soleman S, Yip PK, Duricki DA, Moon LD (2012) Delayed treatment with chondroitinase $\mathrm{ABC}$ promotes sensorimotor recovery and plasticity after stroke in aged rats. Brain 135:1210-1223. CrossRef Medline

Starkey ML, Bartus K, Barritt AW, Bradbury EJ (2012) Chondroitinase ABC promotes compensatory sprouting of the intact corticospinal tract and recovery of forelimb function following unilateral pyramidotomy in adult mice. Eur J Neurosci 36:3665-3678. CrossRef Medline

Steinberg BA, Augustine JR (1997) Behavioral, anatomical, and physiologi- cal aspects of recovery of motor function following stroke. Brain Res Rev 25:125-132. CrossRef Medline

Sunderland A, Tinson DJ, Bradley EL, Fletcher D, Langton Hewer R, Wade DT (1992) Enhanced physical therapy improves recovery of arm function after stroke: a randomised controlled trial. J Neurol Neurosurg Psychiatry 55:530-535. CrossRef Medline

Teasell R, Bitensky J, Salter K, Bayona NA (2005) The role of timing and intensity of rehabilitation therapies. Top Stroke Rehabil 12:46-57. CrossRef Medline

Tennant KA (2014) Thinking outside the brain: structural plasticity in the spinal cord promotes recovery from cortical stroke. Exp Neurol 254:195199. CrossRef Medline

Thallmair M, Metz GA, Z'Graggen WJ, Schwab ME, Raineteau O, Kartje GL (1998) Neurite growth inhibitors restrict plasticity and functional recovery following corticospinal tract lesions. Nat Neurosci 1:124-131. CrossRef Medline

Ueno M, Hayano Y, Nakagawa H, Yamashita T (2012) Intraspinal rewiring of the corticospinal tract requires target-derived brain-derived neurotrophic factor and compensates lost function after brain injury. Brain 135:1253-1267. CrossRef Medline

van den Brand R, Heutschi J, Barraud Q, DiGiovanna J, Bartholdi K, Huerlimann M, Friedli L, Vollenweider I, Moraud EM, Duis S, Dominici N, Micera S, Musienko P, Courtine G (2012) Restoring voluntary control of locomotion after paralyzing spinal cord injury. Science 336:1182-1185. CrossRef Medline

Vavrek R, Girgis J, Tetzlaff W, Hiebert GW, Fouad K (2006) BDNF promotes connections of corticospinal neurons onto spared descending interneurons in spinal cord injured rats. Brain 129:1534-1545. CrossRef Medline

Wade D, Langton-Hewer R, Wood VA, Skilbeck CE, Ismail HM (1983) The hemiplegic arm after stroke. J Neurol Neurosurg Psychiatry 46:521-524. CrossRef Medline

Wahl AS, Schwab ME (2014) Finding an optimal rehabilitation paradigm after stroke: enhancing fiber growth and training of the brain at the right moment. Front Hum Neurosci 8:381. CrossRef Medline

Wahl AS, Omlor W, Rubio JC, Chen JL, Zheng H, Schröter A, Gullo M, Weinmann O, Kobayashi K, Helmchen F, Ommer B, Schwab ME (2014) Asynchronous therapy restores motor control by rewiring of the rat corticospinal tract after stroke. Science 344:1250-1255. CrossRef Medline

Wang Q, Wang PP, Meng PP, Han C, Yue SW (2016) Intensive training accelerates the recovery of motor functions following cerebral ischemiareperfusion in MCAO rats. Eur Rev Med Pharmacol Sci 20:3839-3852. Medline

Watson C, Paxinos G (2006) Rat brain in stereotaxic coordinates. New York: Academic.

Weishaupt N, Li S, Di Pardo A, Sipione S, Fouad K (2013) Synergistic effects of BDNF and rehabilitative training on recovery after cervical spinal cord injury. Behav Brain Res 239:31-42. CrossRef Medline

Winship IR, Murphy TH (2009) Remapping the somatosensory cortex after stroke: insight from imaging the synapse to network. Neuroscientist 15 : 507-524. CrossRef Medline

Winstein CJ, Wolf SL, Dromerick AW, Lane CJ, Nelsen MA, Lewthwaite R, Cen SY, Azen SP (2016) Effect of a task-oriented rehabilitation program on upper extremity recovery following motor stroke: the ICARE randomized clinical trial. Jama 315:571-581. CrossRef Medline

Wolf SL, Winstein CJ, Miller JP, Taub E, Uswatte G, Morris D, Giuliani C, Light KE, Nichols-Larsen D (2006) Effect of constraint-induced movement therapy on upper extremity function 3 to 9 months after stroke: the excite randomized clinical trial. Jama 296:2095-2104. CrossRef Medline

Xu B, Park D, Ohtake Y, Li H, Hayat U, Liu J, Selzer ME, Longo FM, Li S (2015) Role of CSPG receptor LAR phosphatase in restricting axon regeneration after CNS injury. Neurobiol Dis 73:36-48. CrossRef Medline 